Check for updates

Cite this: DOI: 10.1039/c7mh00166e

Received 17th March 2017

Accepted 3rd May 2017

DOI: 10.1039/c7mh00166e

rsc.li/materials-horizons

\title{
Materials and toxicological approaches to study metal and metal-oxide nanoparticles in the model organism Caenorhabditis elegans
}

\author{
Laura Gonzalez-Moragas, (D) ${ }^{a}$ Laura L. Maurer, ${ }^{b}$ Victoria M. Harms, ${ }^{c}$ Joel N. Meyer, \\ Anna Laromaine ${ }^{* a}$ and Anna Roig (D)*a
}

\begin{abstract}
Understanding the in vivo fate and transport of nanoparticles (NPs) is challenging, but critical. We review recent studies of metal and metal oxide NPs using the model organism Caenorhabditis elegans, summarizing major findings to date. In a joint transdisciplinary effort, we highlight underutilized opportunities offered by powerful techniques lying at the intersection of mechanistic toxicology and materials science. To this end, we firstly summarize the influence of exposure conditions (media, duration, C. elegans lifestage) and NP physicochemical properties (size, coating, composition) on the response of the worm to NP treatment. Next, we focus on the techniques employed to study NP entrance route, uptake, biodistribution and fate, emphasizing the potential of extending the toolkit available with novel and powerful techniques. Next, we review findings on several NP-induced biological responses, namely transport routes and altered molecular pathways, and illustrate the molecular biology and genetic strategies applied, critically reviewing their strengths and weaknesses. Finally, we advocate the incorporation of a set of minimal materials and toxicological science experiments that will permit meta-analysis and synthesis of multiple studies in the future. We believe this review will facilitate coordinated integration of both well-established and underutilized approaches in mechanistic toxicology and materials science by the nanomaterials research community.
\end{abstract}

\section{Introduction}

The assessment of nanoparticles (NPs) using Caenorhabditis elegans (C. elegans) has rapidly increased in the last years, supporting its suitability as an in vivo model to screen NPs. ${ }^{1-3}$ C. elegans is a $1 \mathrm{~mm}$ (adult size) worm that lives in soil-associated decaying organic matter. Despite or perhaps because of its simplicity, it is a highly informative animal model widely used in nanotoxicology and biology. ${ }^{4}$ Its ease of maintenance, small size, transparency, short and prolific life cycle, constant cell number, invariant developmental trajectory, highly conserved and well-annotated genome, differentiated anatomical structures and easy genetic manipulation render C. elegans a convenient yet powerful model organism that can be accommodated in any type of research laboratory. ${ }^{5-7}$ As with all model organisms, it has limitations as well, such as the lack of a circulatory system, which limits exposure of distal cells subsequent to feeding, or the absence of specific organs (such as the brain, the

\footnotetext{
${ }^{a}$ Institut de Ciència de Materials de Barcelona, ICMAB-CSIC, Campus UAB, 08193 Bellaterra, Barcelona, Spain.E-mail: alaromaine@icmab.es, roig@icmab.es

${ }^{b}$ ExxonMobil Biomedical Sciences, Inc., Annandale, NJ 08801-3059, USA

${ }^{c}$ Nicholas School of the Environment and Center for the Environmental

Implications of NanoTechnology, Duke University, Durham, NC 27708-0328, USA
}

heart, lungs or the skeletal system). We recently reviewed some of these limitations in more detail. ${ }^{8}$ Overall, C. elegans is a useful model for understanding cellular and molecular processes in vivo (as illustrated for example by the Nobel Prize-winning mechanistic description of apoptosis in C. elegans ${ }^{9}$ ), although extrapolation to organ-level impacts in vertebrates may be challenging.

Environmental (defined here as both ecotoxicological and environmental human health) and biomedical sciences focus on different aspects of nanomaterial research, and often apply different methodological approaches to evaluate NPs in C. elegans (Fig. 1). For instance, in environmental toxicology, C. elegans is used as an aquatic or terrestrial animal model exposed to environmentally relevant concentrations (which are at least 2 orders of magnitude below relevant doses for clinical settings in humans), and outcomes of interest are typically toxicological. In contrast, in nanosciences, C. elegans can be used as a simple model organism to gather preliminary data on the biocompatibility and fate of engineered nanomaterials in any synthetic laboratory. ${ }^{1-3}$ Biomedical studies generally assess a wider range of dose (2-3 orders of magnitude) and have a broad investigation scope including uptake, biodistribution, translocation pathways, as well as molecular mechanisms affected by NP exposure. In addition, C. elegans has also been 


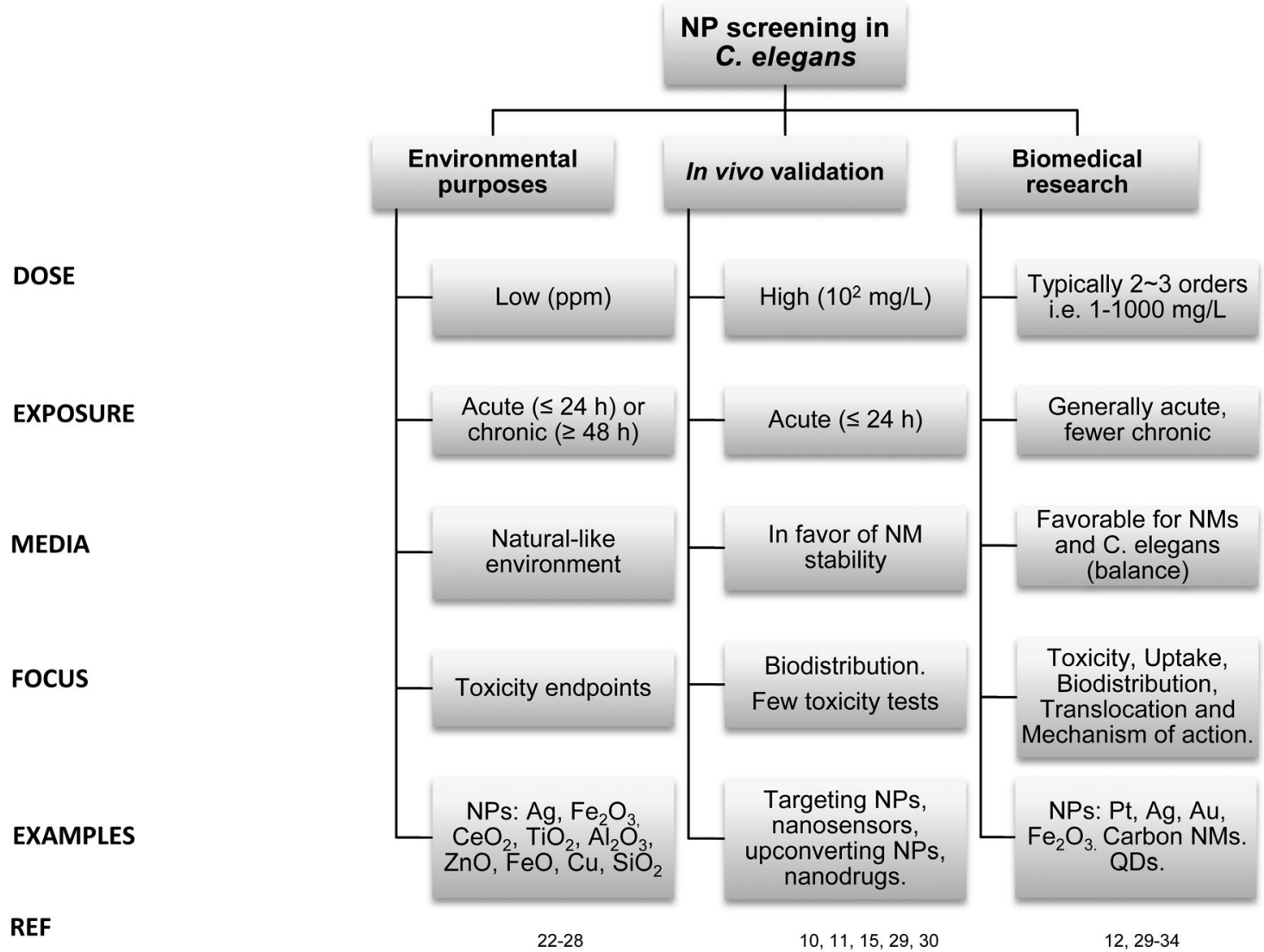

Fig. 1 Main aim and experimental features of recent studies screening nanomaterials in C. elegans. In nanomaterials evaluation, $C$. elegans has been used as an ecotoxicological animal model (these studies are indicated as 'environmental purposes'), and also to assess nanomaterials for biomedical purposes at the initial stages of development (these studies are labeled as 'biomedical research'). Finally, C. elegans also serves as an in vivo platform to validate the efficiency of a nanomaterial for a given application, such as imaging or targeting (application labeled as 'in vivo validation' in the figure). NMs: nanomaterials, NPs: nanoparticles, QD: quantum dots.

used to validate the efficiency of a nanomaterial for a given application in vivo (hence, as a first in vivo proof-of-concept), among them imaging probes, ${ }^{10-12}$ drug delivery systems ${ }^{13,14}$ or targeted agents, ${ }^{15-17}$ without further follow-up of the biological consequences of NP exposure.

Work published to date has provided valuable knowledge and validated techniques to screen the interaction between nanomaterials and C. elegans. However, the great variety in the methodological approaches used (mode of exposure, endpoints measured, etc.) often hinders the comparison of the data gathered and results in what might seem to be contradictory conclusions, especially regarding the potential toxicity of NPs (i.e. effective concentrations, molecular mechanisms, or translocation findings). Harmonization of protocols would enable comparison of the experimental data acquired by different authors and would facilitate a meta-analysis of the existing literature. However, it is unlikely that all researchers will routinely use identical methodologies in initial experiments (screening) of novel materials, as they frequently have different objectives. This is, in some ways, a positive thing, since overreliance on few approaches could blind the research communities to the results of exposures and outcomes (endpoints) not included in the standard methodology. Therefore, we recommend a more practical and strategic approach: to incorporate a suite of basic and highly standardized assays (e.g., $24 \mathrm{~h}$ young adult lethality, $72 \mathrm{~h}$ larval growth, and 3 day reproduction) in all studies, in addition to more specialized and unique research. The outcomes of the standardized assays could then be used as anchors/toxicity markers, allowing direct comparisons between different studies.

The micrometric size of C. elegans enables rapid study of a range of nanomaterials and concentrations by applying a highthroughput set-up able to monitor multiple endpoints in an automated way, for instance integrated in microfluidic platforms. ${ }^{18,19}$ The study of nanomaterials in C. elegans and a diversity of organisms using a collaborative (multi-center) approach can facilitate a cost-effective yet robust evaluation of the risk posed by nanomaterials in the environment and humans. ${ }^{20,21}$

To date, the majority of studies on NPs in C. elegans have focused on noble metal and metal oxide NPs. In the case of metals, silver NPs (Ag-NPs) have been the most investigated. ${ }^{32,33,35-40}$ Fewer efforts have been devoted to other metals such as gold $(\mathrm{Au}-\mathrm{NPs}){ }^{32,41}$ platinum (Pt-NPs) ${ }^{31,42}$ and copper (Cu-NPs). ${ }^{25}$ Published evaluations of metal oxide $\mathrm{NPs}$ include titania $\left(\mathrm{TiO}_{2}-\mathrm{NPs}\right)$, ceria $\left(\mathrm{CeO}_{2}\right.$-NPs), iron oxide $\left(\mathrm{Fe}_{2} \mathrm{O}_{3}-\mathrm{NPs}\right)$, aluminum oxide $\left(\mathrm{Al}_{2} \mathrm{O}_{3}\right.$-NPs), and zinc oxide (ZnO-NPs) nanoparticles, among other compositions. ${ }^{22,24,26-28,34,43-54}$ However, most assays have 
been performed using concentrations expected in soils due to waste disposal of NP-containing products, hence from an environmental/ecological perspective. For instance, ZnO-NPs and $\mathrm{TiO}_{2}$-NPs are used in the cosmetic, food and textile industries; therefore, it is foreseen that some fraction will end up in water and soil during their life cycle. Recently, some reports also included the evaluation of quantum dots or carbon nanomaterials in C. elegans. ${ }^{30,55,56}$

This review, positioned at the interface between the fields of toxicology and materials science, analyzes recent literature reporting the effects of noble metal and metal oxide NPs in C. elegans with the ambition of being useful to researchers from these complementary but sometimes disconnected communities. In the first section, we discuss the importance of exposure parameters especially with respect to NP stability, since aggregation decreases the NP surface area in contact with the worm. We also explore the influence of the physiochemical properties of the NPs on uptake and toxicity in C. elegans. Then, we present current state-of-the-art techniques employed to investigate NP entrance route, uptake, biodistribution and fate, and propose additional techniques to expand the toolkit available. The final section critically reviews the biological responses reported after NP treatment in the worm, including translocation routes, and emphasizes the potential of molecular toxicology and genetic approaches.

\section{Factors influencing nano/bio interactions in C. elegans}

In this section, we review the influence of the exposure conditions (exposure media, C. elegans developmental stage, duration of exposure) and the physicochemical properties of the NPs (size, surface coating, chemical composition) on the effects caused by NPs in C. elegans (Table 1 and Fig. 2).

\subsection{Exposure media}

In the laboratory, C. elegans are typically grown on agar plates of Nematode Growth Media (NGM) with Escherichia coli (E. coli) OP50 as food source. ${ }^{57}$ If NGM agar is mixed with NPs during its preparation, it is difficult to ensure that NPs are evenly distributed in the media since the solid nature of the agar and the high ionic strength of NGM compromise the colloidal stability of the NPs and can lead to their aggregation or precipitation, resulting in a non-homogenous NP exposure to C. elegans. Moreover, the inclusion of living bacteria adds biological surface and active metabolism that can cause uncontrolled and unpredictable effects on the NP status before and during C. elegans exposure (i.e. adsorption of NPs onto the bacterial surface, biotransformation of NPs into subproducts, etc.). The use of alternate media with lower ionic strength might overcome these shortcomings, as they are less likely to induce NP aggregation or metal ion precipitation (if dissolution is important). Among the alternative exposure conditions, some authors have proposed the use of K-agar plates rather than traditional NGM plates, because NGM contains high concentrations of phosphate, which may interact with cations, reducing availability; ${ }^{58}$ acute exposures $(\leq 24 \mathrm{~h})$ in liquid media (typically MHRW or K-medium) in the absence of food; ${ }^{32,59}$ or chronic exposures $(\geq 48 \mathrm{~h}$ ) in liquid media (K-medium or $\mathrm{S}$ basal) with food supplementation. ${ }^{31,33}$ The influence of food, organic matter and ionic strength of the exposure media have also been investigated to better understand more realistic environments that could occur in soil or aquatic environments, given the predominant ecotoxicological focus of most publications. ${ }^{26,35,60,61}$

2.1.1. Exposure in liquid media. The use of liquid media is generally aimed to achieve a homogeneous exposure and maintenance of NP monodispersity during C. elegans treatment. Several liquid media with different ionic strengths are commonly employed in the maintenance of this animal model in the laboratory, among them M9 buffer, S basal or K-medium. ${ }^{57}$ Other recipes commonly used for environmental evaluations include Moderately Hard Reconstituted Water (MHRW), which has low salt content. ${ }^{65}$ In general, media with lower ionic strength favor NP colloidal stability. Conversely, some authors reported formation of micrometric aggregates of Ag-NPs in K-medium that rapidly settled from suspension leading to an elevated effective local "dose" in the bottom of the wells. ${ }^{33,60}$ These events can hinder NP evaluation based on the increase of size up to micrometric size of the tested materials, and may alter NP toxicity. Yang et al. investigated the effect of the ionic strength of the exposure media in Ag-NP toxicity and found that lower ionic strength resulted in greater toxicity; lethal doses were 1.5-12 times higher in MHRW than in K-medium. ${ }^{36}$ They attributed reduced toxicity to NP aggregation due to the subsequent decrease of available surface area for dissolution. ${ }^{36}$ Similarly, Wang et al. exposed nematodes to ZnO-NPs in ultrapure water and K-medium, and observed lower toxicity in the presence of salts, ${ }^{46}$ in good agreement with Donkin and Williams' previous results. ${ }^{66}$

In order to reduce aggregation in liquid media, strategies such as reducing the ionic strength or the exposure time have been employed. Studies prioritizing the colloidal stability of NPs selected an exposure media that favored NP monodispersity and often excluded the presence of food. Ma et al. observed aggregation when $\mathrm{ZnO}$-NPs were diluted in unbuffered K-medium, but not in acetic acid/acetate-buffered K-medium; hence, they used the latter in their experiments. ${ }^{24}$ Gupta et al. followed the same approach. ${ }^{48}$ Roh et al. reported aggregation and precipitation of $\mathrm{CeO}_{2}$ and $\mathrm{TiO}_{2}$-NPs in K-medium at high concentrations, and thus selected exposure to low doses $\left(1 \mathrm{mg} \mathrm{L}^{-1}\right)$ without food for $24 \mathrm{~h}$ to ensure the stability and uniformity of the NP suspensions during the testing period. ${ }^{47}$ Arnold et al. used higher doses of $\mathrm{CeO}_{2}-\mathrm{NPs}$ (2.5-93.75 $\mathrm{mg} \mathrm{L}^{-1}$ ) in MHRW for 3 days; however, they renewed the dosing solutions daily to minimize aggregation effects. ${ }^{50}$ GonzalezMoragas et al. incubated worms in MilliQ water for $24 \mathrm{~h}$ without food ensuring homogenous exposure to a controlled dose of highly stable NPs. ${ }^{34}$ Despite the frequent efforts by the research community to maintain the nano-scale size of the nanomaterials under study, it is arguable that the study of non-dispersed material would be more relevant, as it is the status that will likely occur in the environment or the human body. However, the effort of preserving NP stability and uniformity across the exposure media ensures repeatability of the experiments, and allows discernment of effects 


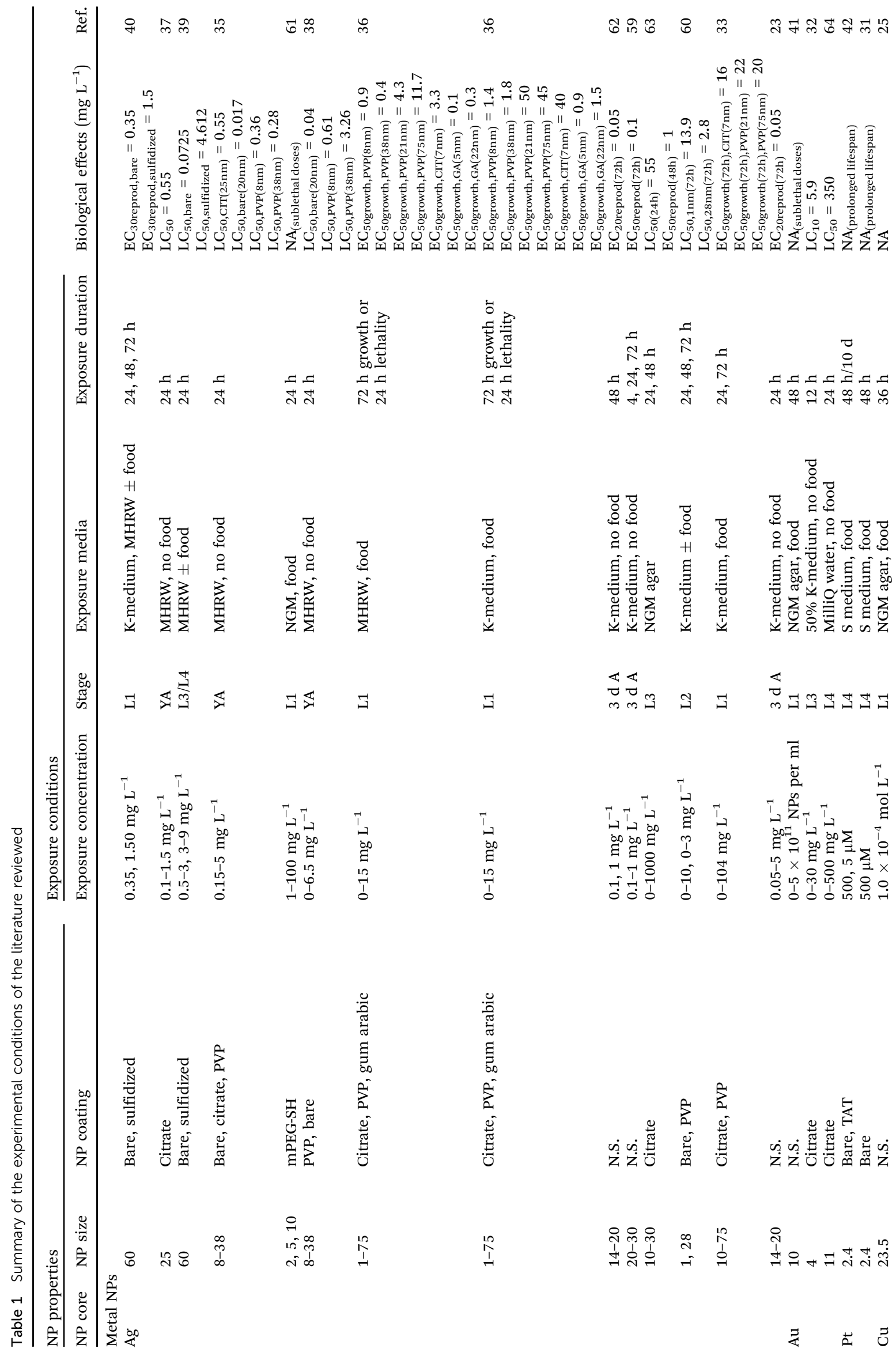




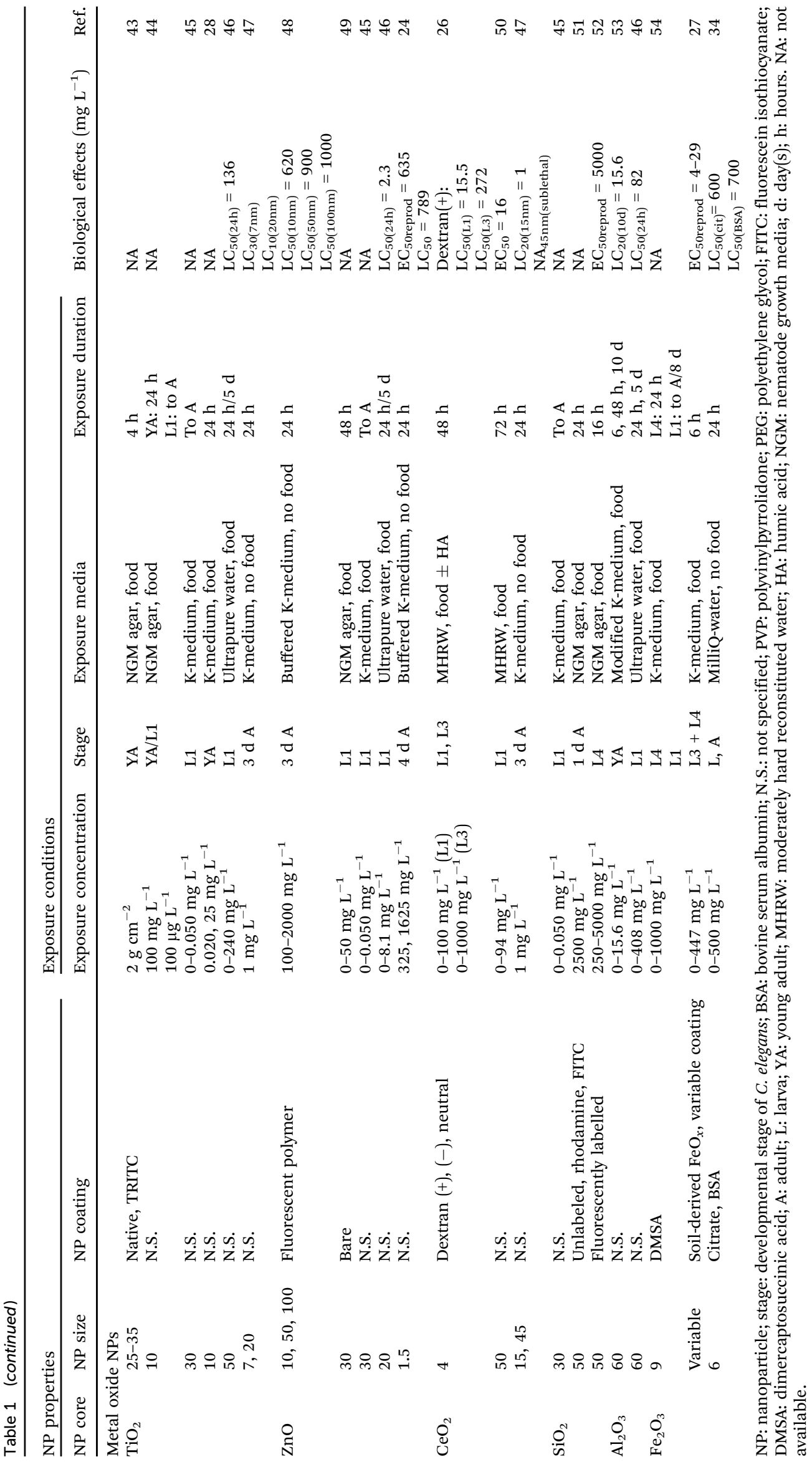




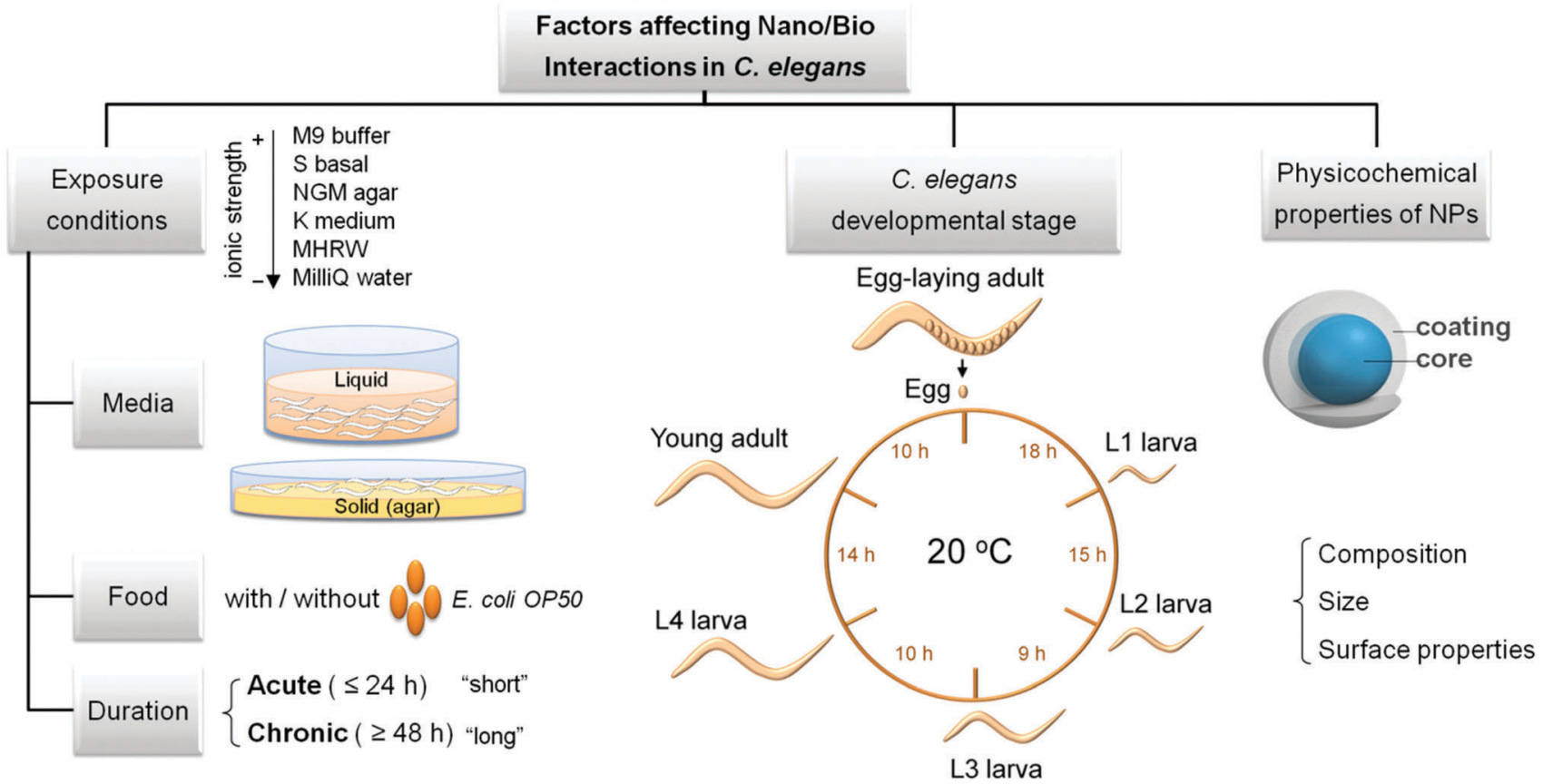

Fig. 2 Factors affecting nano/bio interactions in C. elegans. Parameters to be considered in the experimental settings of NP assessment in C. elegans include exposure conditions and C. elegans developmental stage. The physicochemical properties of NPs such as size, composition and surface properties also determine the biological response of $C$. elegans. The left panel focuses on the exposure conditions. The exposure media can be either liquid or solid, and several recipes with varying ionic strengths and composition are available. Food can be included or excluded during exposure, and exposure duration can range from few hours to the whole lifespan of $C$. elegans. The central panel shows the life cycle of $C$. elegans at $20{ }^{\circ} \mathrm{C}$. The time between developmental stages is indicated in hours. The life cycle progresses through four larval stages (L1-L4) until adulthood. The egg-laying process requires ten additional hours of maturation, and continues for several days. Lifespan under most laboratory conditions is $2-3$ weeks. The right panel schematizes the structure of metal and metal oxide NPs, comprised by an inorganic core and a coating that determine their surface status.

arising solely from the nano-scale properties of the test materials. Ideally, both should be pursued and compared.

2.1.2. Effect of organic components. The addition of food in the exposure system is a parameter of key importance, especially for long-term exposures; most life stages of C. elegans are intolerant to food deprivation over $\sim 24 \mathrm{~h}$ (except the first and dauer larval stages). Several studies have investigated the influence of presence vs. absence of food; however, we identified contradictory findings. Ellegaard-Jensen et al. reported that including $E$. coli in the test medium (K-medium) as a food source increased the toxicity of $1 \mathrm{~nm} \mathrm{Ag-NPs}$ towards nematodes, likely by increasing Ag-NPs bioavailability. ${ }^{60}$ Conversely, Starnes et al. observed decreased mortality after exposure to $60 \mathrm{~nm} \mathrm{Ag-NPs} \mathrm{in} \mathrm{MHRW} \mathrm{in} \mathrm{the} \mathrm{presence} \mathrm{of} \mathrm{food.} \mathrm{The} \mathrm{concen-}$ trations at which C. elegans exhibited equivalent mortality were 4-30 fold higher in the experiment with feeding than without feeding. ${ }^{39}$ Yang et al. also reported strongly mitigated toxicity of $8 \mathrm{~nm}$ PVP-coated Ag-NP in MHRW with natural organic matter (NOM) when food was included. ${ }^{35}$ The reasons for which one group found increased toxicity in the presence of food, while others found decreased or unchanged toxicity, remain unclear. However, the fact that the different groups used NPs of different size, chemical composition, and surface properties, as well as different exposure conditions and toxicity endpoints, could greatly contribute to the variability of their experimental findings. This highlights the potential value of including a set of standardized assays to permit comparison between experiments.

From the environmental perspective, the inclusion of natural organic matter in the exposure system is relevant since C. elegans naturally grows in the decaying organic matter of soil, and many natural water bodies contain significant natural organic matter (NOM). Humic acid (HA) is the most abundant source of NOM in the soil and water. Yang et al. studied the effect of NOM in Ag-NP toxicity and reported the formation of NOM/Ag-NP composites and rescued Ag-NP-induced cellular damage, likely by decreasing intracellular uptake. ${ }^{35}$ Collin et al. exposed nematodes to $\mathrm{CeO}_{2}$ NPs with and without HA as a source of NOM, and observed that HA significantly decreased the toxicity of $\mathrm{CeO}_{2}$-NPs. The authors proposed that the adsorption of HA at the surface of the NPS could form a physical barrier to NP interaction with the cell membrane, reduce binding of NPs to important proteins and biomolecules, and also act as an antioxidant by reacting with ROS and mitigating the oxidative stress induced by $\mathrm{CeO}_{2}-\mathrm{NP}$ exposure. Moreover, the presence of HA greatly influenced Ce bioaccumulation in a manner dependent on the NP/HA ratio: a high ratio increased $\mathrm{Ce}$ accumulation, while a low ratio decreased it, likely due to the negative surface charge of the HA/NP composites. ${ }^{26}$

2.1.3. Controlled exposure in standard conditions. Other authors have chosen exposure in standard culture conditions consisting of NGM agar plates with food. In order to perform 
controlled and reproducible exposures, Pluskota et al. applied NP suspensions to the bacterial lawn and monitored the particle load per area. They studied the dispersity of NPs in suspension by fluorescence correlation spectroscopy (FCS), which showed that single, monodisperse $50 \mathrm{~nm}$ silica ( $\mathrm{SiO}_{2}$-NPs) and polystyrene NPs (PS-NPs) constituted the major mobile fraction. However, they also reported the occurrence of differently sized NP agglomerates in low frequency. ${ }^{52}$ Polak et al. carefully characterized the physicochemical properties of ZnO-NP suspensions in bacteria/ LB mixtures, before pouring them into NGM plates for C. elegans exposure (Fig. 3). In LB broth, $30 \mathrm{~nm}$ ZnO-NPs assembled into $1 \mu \mathrm{m}$ clusters without time-dependent changes in agglomerate levels, suggesting stability of the agglomerates in the test medium. TEM studies showed that the majority of ZnO-NPs formed acicular clusters of few hundred nanometers, resulting in reduced surface charge and thus weaker electrostatic repulsive forces. ZnO-NPs agglomerates did not induce morphological changes or enter the bacteria, but caused the bacteria to secrete extracellular polymeric substances which coated the NPs within $24 \mathrm{~h}$ and could affect the bioavailability of ZnO-NPs. The authors also reported a significant dissolution of the NPs in the exposure media: $\mathrm{Zn}^{2+}$ cations constituted over $50 \%$ of total $\mathrm{Zn}$ after a two day exposure. Therefore, exposure to initially pure ZnO-NPs in vivo in fact represented a mixture exposure of $\mathrm{Zn}^{2+}$ and NPs. However, the authors could not conclude to what extent the observed biological effects were driven by $\mathrm{ZnO}-\mathrm{NPs}$ or by the derived ionic $\mathrm{Zn}{ }^{49}$

\subsection{C. elegans stage and duration of the exposure}

Multiple studies have showed that the extent of NP toxicity depended on the developmental stage of the nematodes at which NP exposure began. Generally, the juvenile forms appear to be more sensitive to NP toxicity, and chronic incubation $(\geq 48 \mathrm{~h})$ is more harmful than shorter exposure periods ( $\leq 24 \mathrm{~h}$ ). For instance, Collin et al. found that L3-larvae were more resistant than L1-larvae to $4 \mathrm{~nm} \mathrm{CeO}_{2}$ exposure. ${ }^{26}$ Zhao et al. also reported that $\mathrm{L} 1$ worms were more sensitive than $\mathrm{L} 4$ individuals or adult nematodes to $10 \mathrm{~nm} \mathrm{TiO}_{2}$-NP toxicity. ${ }^{44}$ The effect of NP treatment also depends on its duration. Wu et al. evaluated the effects of DMSA coated $9 \mathrm{~nm} \mathrm{Fe}_{2} \mathrm{O}_{3}$-NPs in K-medium using three different assay systems: 24 h exposure of L4 nematodes; from L1 to adults ( $\sim 3$ days); and from L1 to 8 day adult. Adverse effects were observed at concentrations higher than $50 \mathrm{mg} \mathrm{L}^{-1}, 0.5 \mathrm{mg} \mathrm{L}^{-1}$ and $0.1 \mathrm{mg} \mathrm{L}^{-1}$, respectively, indicating higher toxicity with increasing treatment duration. ${ }^{54}$ Gonzalez-Moragas et al. investigated the influence of two different surface coatings (citrate and BSA) on $6 \mathrm{~nm} \mathrm{Fe}_{2} \mathrm{O}_{3}$ NPs in larval and in adult populations at several concentrations and found that the BSA coating protected larvae to a greater extent than adults, in good agreement with the more sensitive nature of the juvenile worms. ${ }^{34}$ Furthermore, Zhao et al. found differences in the recovery of C. elegans after acute (24 h; young adults) and chronic (from L1 to adult) exposure to nano-TiO . Chronically-treated nematodes had taken up more NPs than acutely-treated animals, exhibited reduced NP excretion capacity and had endpoints such as length, locomotion or pharyngeal pumping irreversibly altered. ${ }^{55}$

\subsection{Physicochemical properties of the test NPs}

This section describes general findings regarding the influence of NP size, surface coating and composition in the response of C. elegans to NPs. In order to compare the biological effects of different test NPs, toxicological parameters such as $\mathrm{EC}_{50}$ (effect dose $50 \%$ i.e. for growth or reproduction) or $\mathrm{LC}_{50}$ (lethal dose $50 \%$ ) can be employed (Table 1). However, the conditions of

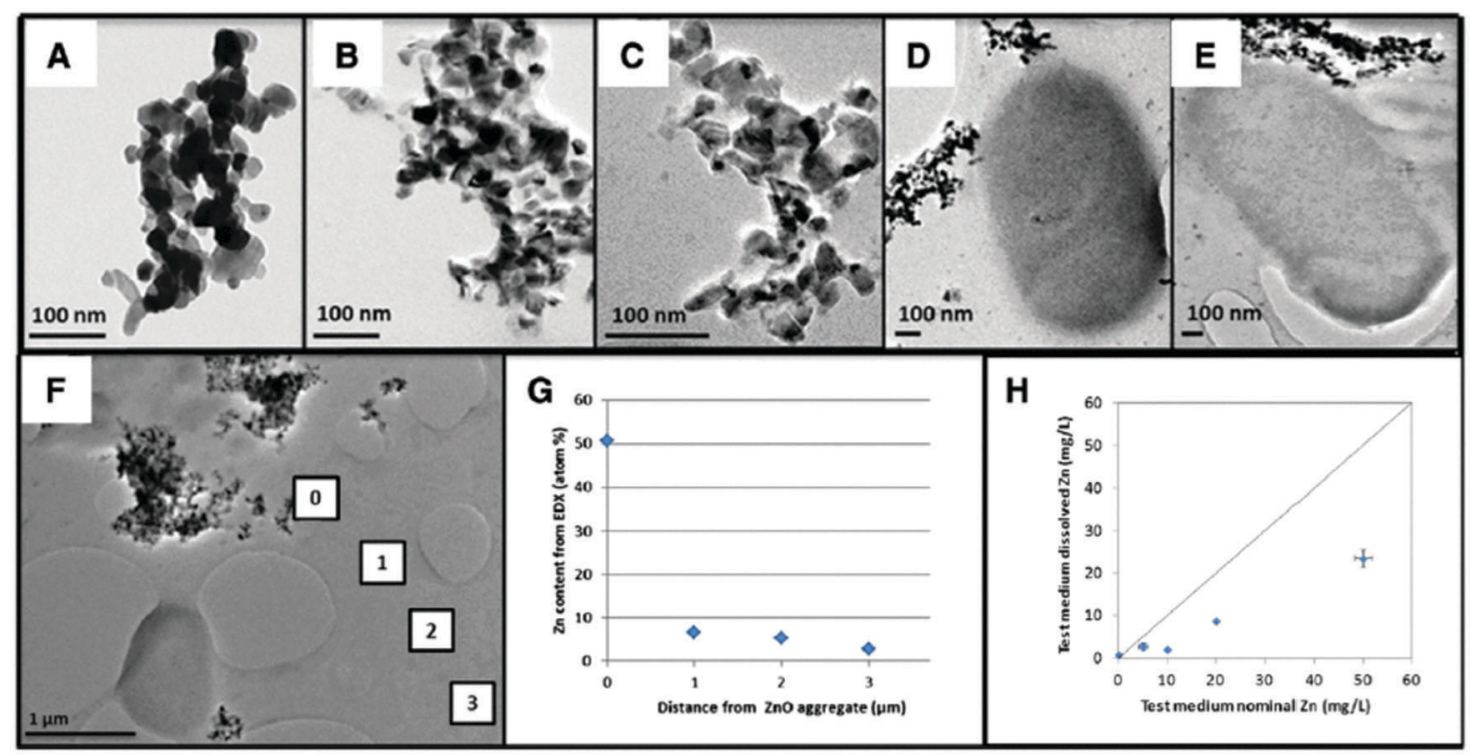

Fig. 3 Transmission Electron Microscopy (TEM) of ZnO-NPs in the exposure media. Images of ZnO-NPs in: (A) HPLC water, (B) LB-broth at 0 h, (C) LBbroth at $24 \mathrm{~h}$, (D) LB-broth with bacteria (OP50) at $0 \mathrm{~h}$, (E) LB-broth with bacteria (OP50) at $24 \mathrm{~h}$. (F) Energy-dispersive X-ray spectroscopy (EDX) analyses and chemical mapping on four micron-sized windows were performed to determine: $(G)$ the percentage of $\mathrm{Zn}$ content, $(H)$ the relative dissolution rate quantified (H). Adapted from Polak et al. ${ }^{49}$ 
exposure must be considered in the interpretation of these values.

2.3.1. Size. Most nanotoxicological studies agree on the higher toxicity of small NPs. Meyer et al. reported more intracellular uptake of small Ag-NPs (10 and $21 \mathrm{~nm}$ ) than larger particles $(75 \mathrm{~nm})$ in K-medium. ${ }^{33}$ Among their biological effects, growth inhibition was reported for all the tested NPs but bagging and intergenerational transfer was solely observed for $10 \mathrm{~nm}$ Ag-NP, suggesting that Ag-NP uptake and reprotoxicity are size-dependent. Contreras et al. compared the uptake of 2, 5 and $10 \mathrm{~nm}$ PEG-coated Ag-NPs and found that a lesser amount of $\mathrm{Ag}$ was internalized in C. elegans exposed to small $\mathrm{Ag}$ particles compared with larger particles, probably due to the increased excretion of the smaller NPs. They also reported sizedependent effects on lifespan and fertility after exposure for multiple generations, but no size-dependence of body length and motility. ${ }^{61}$ Ellegaard-Jensen et al. observed higher lethality of $28 \mathrm{~nm}$ PVP Ag-NPs than $1 \mathrm{~nm}$ bare Ag-NPs and attributed it to a combination of effects of coating, Ag-solubility and higher uptake rates. They proposed that larger particle size may enable faster uptake rates by oral ingestion and thus higher mass doses than exposure to smaller stable particle sizes. ${ }^{60}$ Ahn et al. also observed reduced toxicity of PVP-Ag-NPs compared to bare Ag-NPs, and higher toxicity for the smaller NPs. ${ }^{38}$ Yang et al. evaluated Ag-NPs of 1-75 nm and observed no relationship between the growth inhibition and the diameter of Ag-NP, but a linear correlation between Ag-NP toxicity and dissolved silver. ${ }^{36}$ Taken together, these studies suggest a rough trend towards size-dependent uptake, reprotoxicity and lifespan of Ag-NPs, but no effect of size in locomotion behavior and body length. It is likely that inconsistencies relate at least in part to other variables (functionalization, etc.). Gonzalez-Moragas et al. recently reported higher toxicity, in terms of survival and brood size, of $11 \mathrm{~nm}$ Au-NPs compared to Au-NPs of $150 \mathrm{~nm}$ after $24 \mathrm{~h}$, but no size-dependent effects on body length. ${ }^{67}$

In a study of metal oxide NPs, Roh et al. reported differences in toxicity endpoints (growth, fertility and survival) depending on the size of $\mathrm{CeO}_{2}$-NPs and $\mathrm{TiO}_{2}$-NPs: the toxicity exhibited by the smaller sized NPs $(7,15 \mathrm{~nm})$ was higher than that observed for the larger sized ones $(20,45 \mathrm{~nm}) .{ }^{47}$ Gupta et al. also observed higher toxicity for small ZnO-NPs $(10 \mathrm{~nm})$ than larger-sized particles (50 and $100 \mathrm{~nm})$, especially at high doses $\left(\geq 700 \mathrm{mg} \mathrm{L}^{-1}\right) .{ }^{48}$

2.3.2. Surface properties. Surface coating can significantly affect NP toxicity by modulating NP uptake, bioavailability and reactivity; hence, its engineering can be used as a strategy to gain control over the nano/bio interactions and prevent undesired post-synthesis modifications either in the environment or in the human body. Yang et al. studied citrate, PVP and gum arabic as surface coatings of small Ag-NPs $(<10 \mathrm{~nm})$ and found coating-dependent effects: gum arabic was $\sim 9$-fold more toxic than PVP, which in turn was $\sim 3$-fold more toxic than citrate. The authors found that the most toxic Ag-NPs were also the most soluble. Starnes et al. investigated the effect of Ag-NP sulfidation, a major transformation occurring in the wastewater treatment process, and reported reduced bioavailability, lower toxicity and distinct toxicity mechanisms of sulfidized Ag-NPs compared to bare particles. ${ }^{39,40}$ Surface engineering can also result in enhanced bioavailability; e.g., Kim et al. conjugated nano-Pt with HIV-1 TAT fusion protein, a cell-penetrating peptide, which resulted in an antioxidant activity 100 times higher than unconjugated Pt-NPs. ${ }^{42}$

Collin et al. studied the effect of surface charge using $4 \mathrm{~nm}$ dextran-coated $\mathrm{CeO}_{2}$-NPs and concluded that NP toxicity and accumulation in tissues and organs depended on NP surface properties. Positively charged $\mathrm{CeO}_{2}$-NPs were significantly more toxic to $C$. elegans and bioaccumulated to a greater extent than neutral and negatively charged NPs. The latter NPs mainly accumulated in the gut, while positively charged $\mathrm{CeO}_{2}-\mathrm{NPs}$ were also detected throughout the $C$. elegans body. The authors related the higher cytotoxicity of the positively charged NPs to higher cellular uptake, and also due to the direct interaction of cationic NPs with cells which could disrupt the cell membrane's lipid bilayer. ${ }^{26}$ Surface charge also affected the oxidation state of $\mathrm{Ce}$ in the C. elegans tissues after uptake: greater reduction of $\mathrm{Ce}$ from $\mathrm{Ce}(\mathrm{IV})$ to $\mathrm{Ce}(\mathrm{III})$ was found in C. elegans when exposed to the neutral and negatively charged relative to positively charged $\mathrm{CeO}_{2}$-NPs. The $\mathrm{Ce}$ reduction suggests oxidative damage of macromolecules or generation of ROS. Interestingly, the authors also showed that coating $\mathrm{CeO}_{2}$-NPs with NOM at environmentally realistic ratios of $\mathrm{HA}$ to $\mathrm{CeO}_{2}$ reduced the effects of initial surface status, and rendered positively charged $\mathrm{CeO}_{2}-\mathrm{NPs}$ significantly less toxic. $^{26}$

Höss et al. investigated the toxicity of soil-derived colloidal iron oxides $\left(\mathrm{FeO}_{x}\right)$ with variable aggregate size and variable association with NOM, and found that the toxicity was dependent on aggregate size and specific surface area (Fig. 4A), with differences up to 7-fold in their toxic concentrations. $\mathrm{FeO}_{x}$ associated with HA or citrate were less toxic than NOM-free colloids. In contrast, ferrihydrite containing proteins and polysaccharides from mobile NOM was even more toxic than NOMfree ferrihydrite of similar aggregate size. ${ }^{27}$ This study reinforces the importance of NOM as a determinant of the ecological risks posed by nanomaterials. On the biomedical side, GonzalezMoragas et al. reported lower toxicity of albumin-coated $\mathrm{Fe}_{2} \mathrm{O}_{3}$ NPs compared to citrate-stabilized $\mathrm{Fe}_{2} \mathrm{O}_{3}$-NPs, and attributed it to the reduced interaction of the former with the $C$. elegans cells, confirming the impact of a controlled surface coating on the bioidentity of NPs in vivo. ${ }^{34,68}$

2.3.3. Chemical composition. Pluskota et al. assessed fluorescently labeled $50 \mathrm{~nm} \mathrm{SiO}_{2}$-NPs and PS-NPs and concluded that their translocation in C. elegans was dependent on composition (Fig. 4B). $\mathrm{SiO}_{2}$-NPs were exclusively found in primary organs of entry, e.g. the lumen of the digestive tract, while carboxy PS-NPs translocated to secondary organs and also to the cytoplasm of early embryos. ${ }^{52} \mathrm{Wu}$ et al. also compared the toxicity of $\mathrm{TiO}_{2}, \mathrm{ZnO}-\mathrm{NPs}$ and $\mathrm{SiO}_{2}$-NPs with the same nanosize $(30 \mathrm{~nm})$, and reported differences in their toxicity solely due to the different composition. The toxicity order was: ZnONPs $>\mathrm{TiO}_{2}$-NPs $>\mathrm{SiO}_{2}$-NPs, using growth, locomotion behavior, reproduction, and ROS production as endpoints. ${ }^{45}$ GonzalezMoragas et al. reported higher toxicity of Au-NPs of $11 \mathrm{~nm}$ 
A aggregate size / presence + composition of associated OM

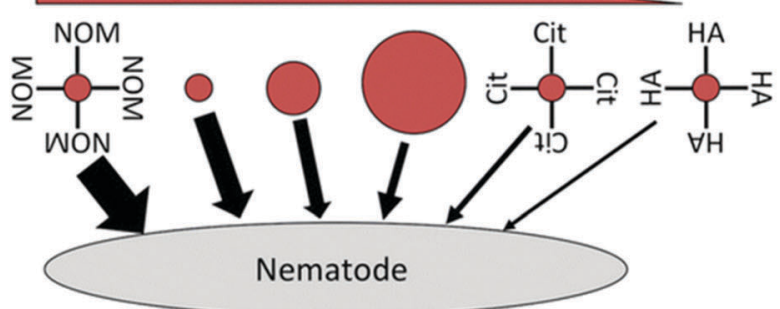

B

\begin{tabular}{llll}
\hline organ/tissue & silica-NPs & polystyrene-NPs & $\begin{array}{l}\text { carboxy } \\
\text { polystyrene-NPs }\end{array}$ \\
\hline pharynx - lumen & + & + & + \\
intestine - lumen & + & + & + \\
intestine - tissue & - & + & + \\
proximal gonad & - & + & + \\
spermatheca & - & - & + \\
early embryo/cytoplasm & - & - & + \\
\hline
\end{tabular}

Fig. 4 Effect of NP size, surface coating and chemical composition on C. elegans toxicity and biodistribution. (A) Scheme of the effect of aggregate size and presence of NOM in the toxicity of soil-derived colloidal iron oxide in C. elegans. Adapted from Höss et al. ${ }^{27}$ (B) Table indicating the distribution of silica, polystyrene and carboxy-polystyrene NPs within the body of treated C. elegans. Adapted from Pluskota et al. ${ }^{52}$

compared to $\mathrm{Fe}_{2} \mathrm{O}_{3}$-NPs of similar size and surface properties, as illustrated by their $\mathrm{LD}_{50}$ values of $350 \mathrm{vs.} 600 \mu \mathrm{g} \mathrm{ml} \mathrm{m}^{-1}$, respectively, in young adults. ${ }^{34,64,67}$ The authors attributed these differences to the essential biological role of iron in C. elegans and the nonnatural presence of gold in organisms. $C$. elegans have a robust iron homeostasis capacity that is able to deal with significant iron overload; however they presumably lack any specific response to overcome the stress generated by biopersistent gold NPs.

Besides metal and metal oxide NPs, quantum dots ${ }^{69}$ and carbonbased nanomaterials such as graphene oxide have been widely studied recently. ${ }^{70,71}$ Their molecular effects are briefly reviewed in the Section 4.4, since they are out of the main scope of this review.

\section{Techniques to investigate entrance route, uptake, biodistribution and fate}

Nanopharmacokinetics are critical for both environmental and biomedical research. Based on the small size of the materials under study, techniques with spatial resolution at the nanoscale are required to discern and identify particles with certainty at multiple biological levels, from the organismal down the intracellular scales, in order to shed light on single-NP localization, translocation routes and NP status in vivo. In C. elegans, entrance of metal and metal oxide NPs has been reported to occur mainly through the alimentary system, consistent with the fact that $C$. elegans does not discern between entities up to $5 \mu \mathrm{m}$ when feeding. ${ }^{72}$ The most prevalent techniques applied to date to determine NP uptake and fate in this animal model have been fluorescent microscopy, ${ }^{42,51,52}$ hyperspectral dark-field microscopy, ${ }^{33,38}$ and to a much lesser extent, transmission electron microscopy (TEM), ${ }^{53,68}$ synchrotron-based techniques ${ }^{25}$ and other analytical techniques. ${ }^{27,43}$ In this section, we illustrate how the entrance route, uptake, biodistribution and fate of metal and metal oxide NPs have been evaluated in C. elegans, and propose to extend the toolkit of available techniques.

\subsection{Fluorescence microscopy}

By using fluorescence microscopy Pluskota et al. showed that fluorescently labeled $50 \mathrm{~nm}$ NPs (PS and $\mathrm{SiO}_{2}$ ) were efficiently ingested by the worms during feeding, and translocated to primary organs such as epithelial cells of the intestine, as well as to secondary organs belonging to the reproductive tract. Within the intestine, NPs accumulate with decreasing concentrations from the anterior to the posterior regions of the intestine. Cytoplasmic uptake of $50 \mathrm{~nm}$ PS-NPs was observed in early embryos. ${ }^{52}$ Scharf et al. identified two entry portals of silica and PS-NPs: via the pharynx to the intestinal system, and via the vulva to the reproductive system. Using light sheet microscopy, they identified NPs throughout the cytoplasm and the cell nucleus in single intestinal and vulval cells (Fig. 5). ${ }^{51}$ Gupta et al. investigated the biodistribution of 10, 50, $100 \mathrm{~nm}$ ZnO-NPs conjugated with a fluorescent polymer, and reported that the smaller NPs showed a uniform distribution of fluorescence in a wide range of cells and tissues including a large number of eggs, whereas 50 and $100 \mathrm{~nm}$ ZnO-NPs were only recorded at particular points either in anterior or posterior intestinal regions. ${ }^{48}$ However, it is important to note that fluorescence microscopy is limited to a spatial resolution of $200 \mathrm{~nm}$, hence, without the use of complementary techniques, the possibility that single NPs penetrate further into C. elegans tissue or are taken up intracellularly cannot be excluded.

\subsection{Hyperspectral microscopy}

Meyer et al. studied the biodistribution of citrate and PVP-coated Ag-NPs (10-75 nm) using hyperspectral microscopy and observed that all the NPs tested were internalized by the intestinal cells, but only the citrate-coated Ag-NPs detectably transferred to the germ line (Fig. 5). ${ }^{33}$ Yang et al. reported that the majority of Ag-NPs $(8-38 \mathrm{~nm})$ were located in the digestive tract, and detected limited tissue uptake by hyperspectral microscopy but not by TEM. ${ }^{35}$ Arnold et al. also applied hyperspectral imaging to study the localization of $50 \mathrm{~nm}$ $\mathrm{CeO}_{2}$-NPs and detected NPs both in the intestinal tract and on the surface of the worm, but not inside the intestinal cells. ${ }^{50}$

\subsection{Transmission electron microscopy}

TEM has sufficient spatial resolution to allow single-NP detection. It has been applied to investigate the integrity of the intestinal barrier in NP-treated worms, and also to study the intracellular location of internalized metal and metal oxide NPs including $\mathrm{TiO}_{2}, \mathrm{Fe}_{2} \mathrm{O}_{3}$ and $\mathrm{Au}$ (Fig. 6). ${ }^{35,53,68,73}$ TEM was also used to investigate the potential for recovery of $\mathrm{TiO}_{2}-\mathrm{NP}$ treated 


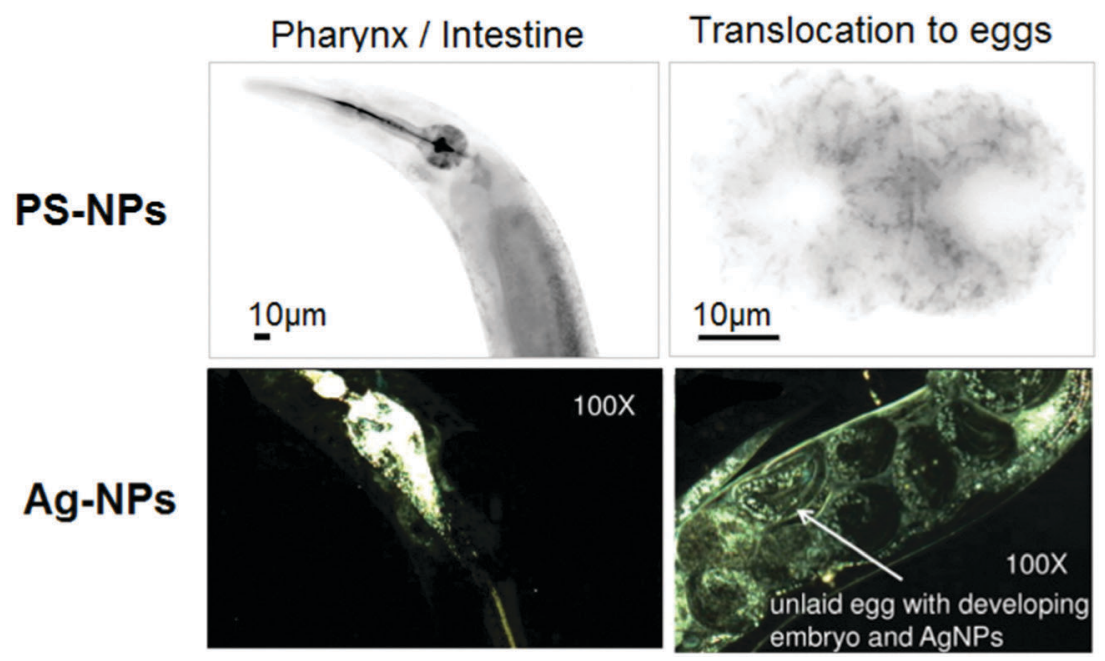

Fig. 5 Use of fluorescence and hyperspectral imaging to characterize NP pharmacokinetics. Worms fed on a bacterial together with PS-NPs and AgNPs. Upper panels show epifluorescence images of carboxy $50 \mathrm{~nm}$ PS-NPs in the intestine (left) and cytoplasm of early embryos (right). Adapted from Scharf et al. ${ }^{51}$ Lower panels present hyperspectral images showing $10 \mathrm{~nm}$ citrate Ag-NPs in the intestine (left) and transference to the offspring (right). AgNP identity was confirmed by hyperspectral analysis. Adapted from Meyer et al. ${ }^{33}$

nematodes; the intestinal barrier of acutely-treated worms was able to recover, in contrast to the lasting defects induced after prolonged exposure. ${ }^{44}$ It can also provide further clues about NP translocation routes, i.e. by endocytosis, although this should be confirmed by chemical identification or molecular mechanistic evidence. ${ }^{68,74}$ However, applying protocols for optimal sample preparation is crucial to minimize technical difficulties such as the worms' orientation. Sampling at random locations along the body of the worm can limit the information obtained by TEM visualizations; moreover, the analysis of a large number of sections is very costly and laborious. To maximize the control of the anatomical area investigated in the cross-sections, targeted ultramicrotomy protocols can be applied using correlated light and electron microscopy (CLEM), with the aim of establishing a statistically significant and biologically meaningful link between the location in the body and the NP status in vivo. ${ }^{75}$

\subsection{Scanning electron microscopy}

Scanning electron microscopy (SEM) allows investigation of the morphology of the C. elegans external surface, the cuticle, in detail. Kim et al. explored the dermal effects of NP exposure in C. elegans using SEM. ${ }^{63}$ After a $24 \mathrm{~h}$ exposure to citrate coated $10 \mathrm{~nm}$ Ag-NPs in NGM agar, they observed severe epidemic edema and bursting of the cuticle of C. elegans (Fig. 7A-C), suggesting that Ag-NPs can induce adverse physical effects via the dermal route. Given that previous studies in liquid ${ }^{33}$ did not reveal such effects, the authors proposed they were induced by the movement of $C$. elegans in the agar plates where the $\mathrm{Ag}$-NPs were distributed. More recently, we evaluated the external surface of worms treated with $\mathrm{Fe}_{2} \mathrm{O}_{3}$ and Au-NPs in liquid by SEM coupled to energy-dispersive X-ray spectroscopy (EDX), an elemental analysis technique that enables the study of chemical composition. We could not visualize NPs on the cuticle of C. elegans by SEM, nor did we detect the presence of iron or gold elements on this structure by EDX (Fig. 7D-F) after thorough rinsing of the treated worms. These results are in good agreement with the well-accepted notion that the main uptake route for metals and metal oxide NPs into the tissues of the worm is the gut, and that nanotoxicity is mainly attributed to the NP feeding in C. elegans. ${ }^{33,76,77}$

\subsection{Synchrotron and microprobe techniques}

Among the synchrotron techniques, synchrotron radiation $\mathrm{X}$-ray fluorescence ( $\mu$-SRXRF) has been used to map the metal distribution in $C$. elegans, while synchrotron X-ray absorption near-edge spectroscopy ( $\mu$-XANES) has provided information regarding the oxidation state and coordination environment of metals. ${ }^{25,43}$ The combination of $\mu$-SRXRF and $\mu$-XANES is a powerful tool to study the subcellular distribution and chemical species of metal and metal NPs of interest. Using $\mu$-SRXRF, Gao et al. showed that $24 \mathrm{~nm}$ Cu-NP exposure resulted in elevation of $\mathrm{Cu}$ and $\mathrm{K}$ levels in the C. elegans body, and also in changes in $\mathrm{Cu}$, $\mathrm{Fe}$ and $\mathrm{Zn}$ biodistribution (Fig. 8). However, $\mathrm{Cu}^{2+}$ exposure resulted in a much higher absorption and accumulation. ${ }^{25}$ Regarding the use of nuclear microprobe techniques, Le Trequesser combined scanning transmission ion microscopy (STIM) and micro-proton-induced X-ray emission ( $\mu$-PIXE) to detect and quantify $30 \mathrm{~nm} \mathrm{TiO}_{2}$-NPs in C. elegans. After $4 \mathrm{~h}$ exposure, NPs were visible only in the lumen of the alimentary system extending from the pharynx to the anal region, and were retained there even $24 \mathrm{~h}$ after feeding. ${ }^{43}$ Given that alterations in the distribution of trace metal such as $\mathrm{Fe}, \mathrm{Cu}, \mathrm{Zn}$ or $\mathrm{Mn}$ are sometimes related to certain pathological states, the use of these techniques is of value in the study of alterations in metal homeostasis.

\subsection{Analytical chemistry techniques}

Among the analytical techniques applied to investigate NPs in C. elegans, different micro-spectroscopy modalities have been used to characterize NP status, while quantitation of NP uptake 

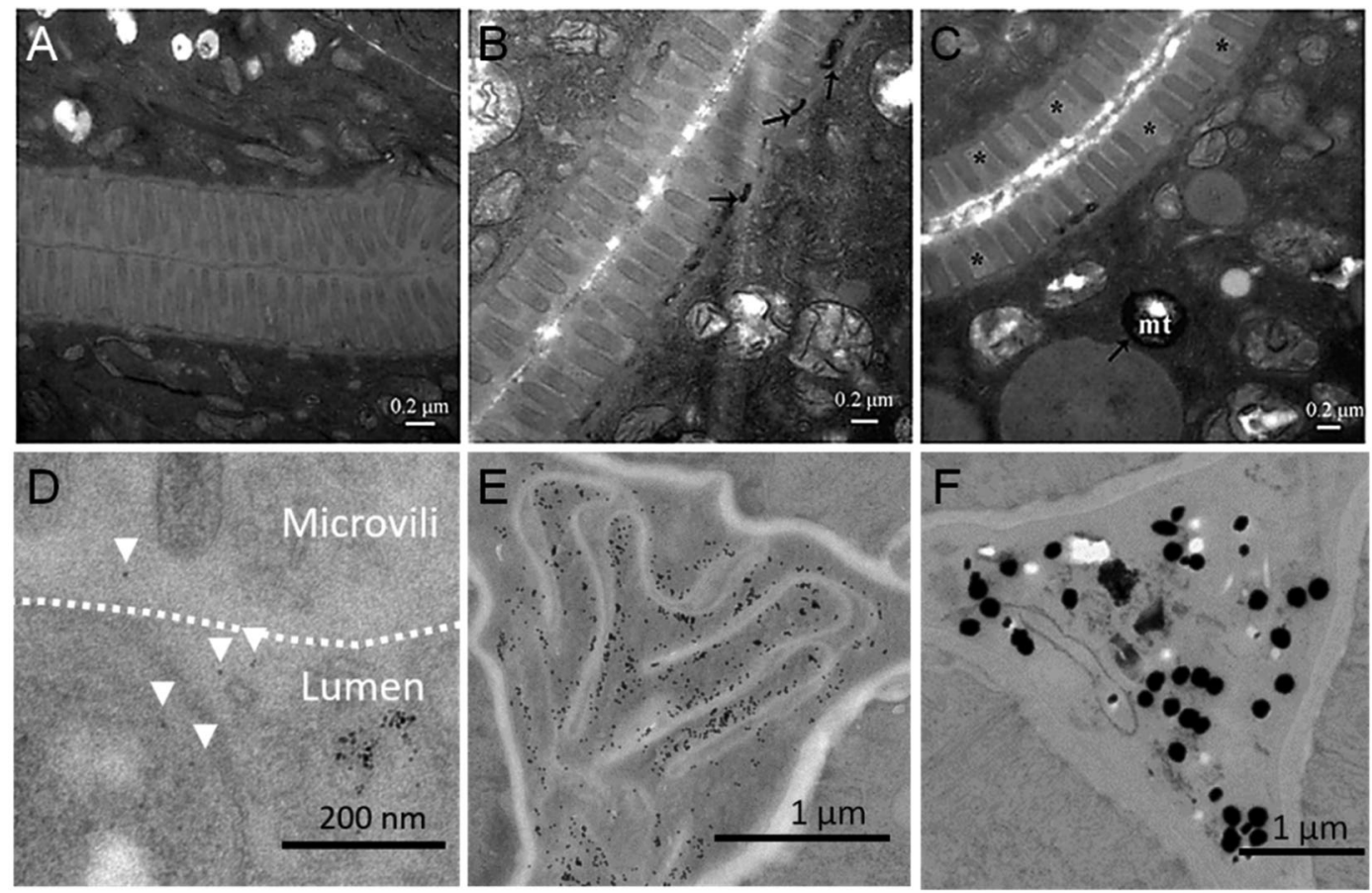

Fig. 6 Use of TEM to characterize NP kinetics and dynamics in C. elegans. (A-C) Ultrastructural changes of intestine and uptake in TiO $\mathrm{O}_{2}-\mathrm{NPs}$ exposed nematodes after transfer to control conditions. (A) Unexposed nematodes. (B) Nematodes exposed to $100 \mathrm{mg} \mathrm{L}^{-1} \mathrm{TiO}_{2}-\mathrm{NPs}_{\mathrm{immediately}}$ upon transfer to control conditions. (C) Nematodes exposed to $100 \mu \mathrm{g} \mathrm{L}-1 \mathrm{TiO}_{2}-\mathrm{NPs}$ after $48 \mathrm{~h}$ in standard conditions. Asterisks indicate positions where microvilli are absent. Arrowheads indicate the location of $\mathrm{TiO}_{2}-\mathrm{NPs}$. Mitochondria, mt. Adapted from Zhao et al. ${ }^{44}$ (D) TEM image of Fe $\mathrm{O}_{3}-\mathrm{NP}$ treated young nematodes, showing individual NPs in close contact with the microvilli (arrowheads) within the glycocalyx, delimited with a dotted line. Adapted from Gonzalez-Moragas et al. ${ }^{68}$ (E and F) TEM image of the pharynx of C. elegans treated with (E) $11 \mathrm{~nm}$ Au-NPs and (F) $150 \mathrm{~nm}$ Au-NPs, prepared following the targeted ultramicrotomy protocol. Adapted from Gonzalez-Moragas et al. ${ }^{64}$

has been mainly addressed by chemical elemental analysis (ICP-MS). Polak et al. used Raman microspectroscopy to identify differences in biomolecular composition and quantify changes in internal $\mathrm{Zn}$ load within individual nematodes, either wild-type (N2 strain) or metal sensitive (triple knockout mutants $m t l-1$; $m t l-2 ; p c s-1$ ) (Fig. 9A). A significant separation of the spectra was observed in the head and tail region of the mutants upon exposure to ZnO-NPs compared to wild-type nematodes, confirming that the phenotype of the metallochaperone mutant is more affected by ZnO-NP exposure. Exposure of $m t l-1 ; m t l-2 ; p c s-1$ nematodes to ZnO-NPs caused reductions in peak intensities of proteins, amino acids (cytochrome $c$, amide I, phenylalanine) and nucleic acids, highlighting a broad effect on the nematode biology. ${ }^{49}$ Höss et al. investigated the accumulation of soilderived ferrihydrites using the ferrozine assay for iron determination. They detected relatively high Fe concentrations after a $6 \mathrm{~h}$ exposure $\left(2 \mu \mathrm{g} \mathrm{mg}^{-1}\right.$ worm), however Fe uptake decreased after $2 \mathrm{~h}$ under normal conditions due to defecation of the NPs contained in the intestinal lumen (disposal of $50 \% \mathrm{Fe}$ ), and it was further reduced by the disposal of the surface-attached Fe during molting (additional $80 \%$ reduction of nematodeassociated Fe) (Fig. 9B). Overall, the Fe concentration in the tissue of exposed C. elegans was 4.5 times higher than in the control animals, however, the ferrozine test did not provide information of the form of iron. Therefore, Fe uptake could represent intact ferrihydrites colloids but also iron ions released under the mildly acidic conditions of the C. elegans gut (Fig. 9C). ${ }^{27}$ More recently, Johnson et al. applied ICP-MS to quantify Au-NP uptake by C. elegans and, operating in single particle ICP-MS mode, to characterize Au-NP status inside the animals. The authors emphasized the reliability of the quantitative protocol and its potential to investigate NP biotransformation in the gut. ${ }^{78}$ However, the requirement of NP extraction from treated C. elegans (by means of solvents) may have an effect on NP status, limiting the information that can be provided by this protocol. Conversely, TEM allows the in situ characterization of Au-NP size and aggregation status inside the intestine of NP-treated C. elegans. ${ }^{64}$

\subsection{Other experimental techniques with potential to characterize nano/bio interactions}

The characterization of NP status inside C. elegans (that is, NP size, aggregation and properties in vivo) has been relatively minimal; hence there are only few studies devoted to how ingestion by C. elegans alters the NPs (i.e. formation of protein corona, digestion in the gut, etc.). ${ }^{24,33,34}$ The adoption of additional materials science techniques in combination with biological evaluations could contribute to a more solid evaluation of inorganic nanomaterials in C. elegans. We propose below some possibilities that could permit advance in this direction.

3.7.1. Advanced microscopy coupled to micro-spectroscopy. The combination of microscopy and spectroscopy can contribute to comprehensively understand nano/biological interactions. 
A
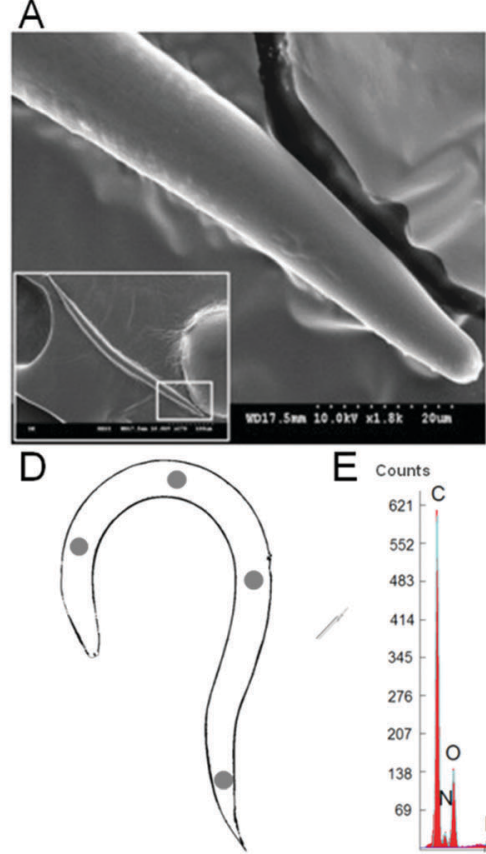

$\mathrm{E}$$$
\begin{aligned}
& 621 \\
& 552 \\
& 485 \\
& 4 \\
& 3 \\
& 2 \\
& 2 \\
& 1 \\
& 1 \\
& 6
\end{aligned}
$$

B
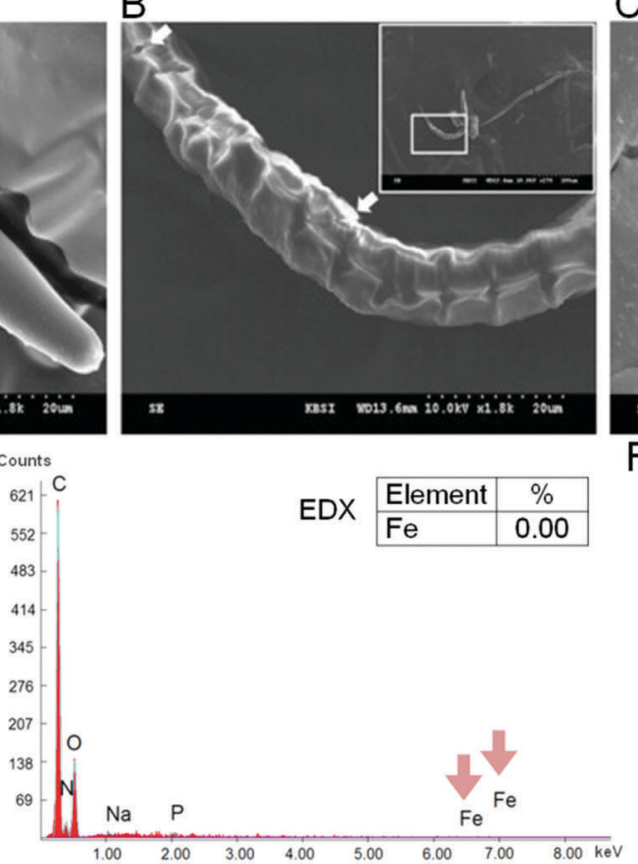
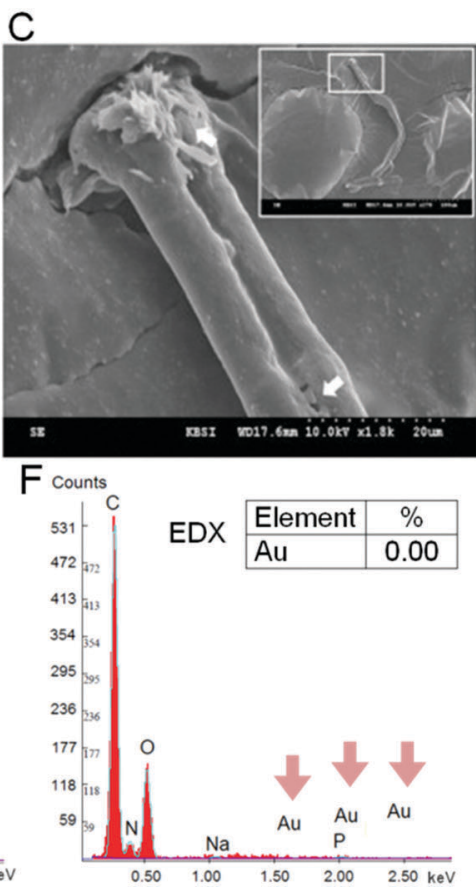

Fig. 7 Investigation of the interaction between NPs and the external surface of C. elegans by SEM. (A-C) Scanning electron micrograph of $C$. elegans exposed to citrate Ag-NPs: (A) control, (B) $10 \mathrm{mg} \mathrm{L}^{-1}$, and (C) $100 \mathrm{mg} \mathrm{L}^{-1}$. The white arrows indicate epidermal divisions and necrosis. Adapted from Kim et al. ${ }^{63}$ (D-F) SEM-EDX analysis of (E) $\mathrm{Fe}_{2} \mathrm{O}_{3}-\mathrm{NP}$ treated C. elegans and (F) Au-NP treated C. elegans. Different locations of the body of treated animals were analyzed by EDX, as schematized in panel D. Adapted from Gonzalez-Moragas et al. ${ }^{34,64}$

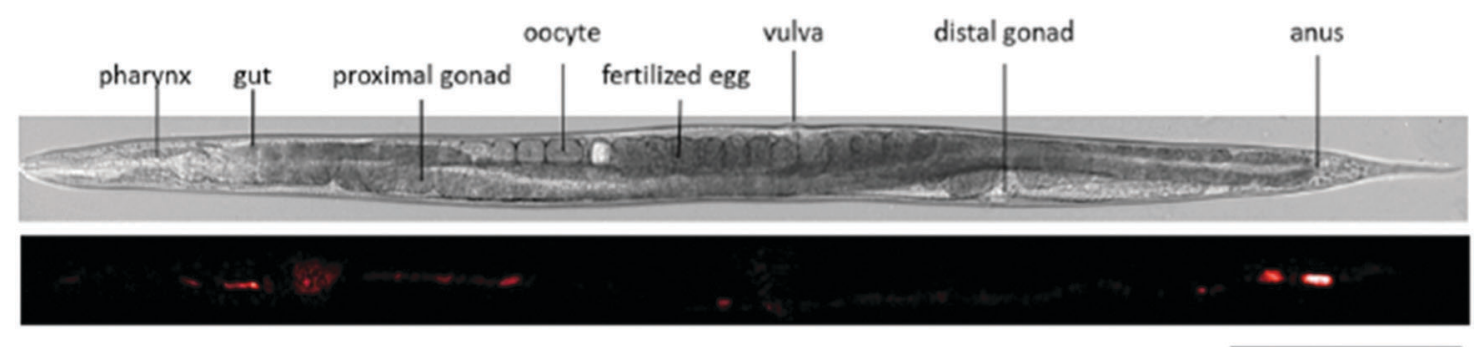

Fig. 8 Use of $\mu$-PIXE to characterize NP toxicokinetics in C. elegans. Above, synchronized worm observed by conventional light microscopy, indicating the different anatomical structures. Below, $\mu$-PIXE maps of titanium in C. elegans body. Scale bar, $150 \mu \mathrm{m}$. Adapted from Le Trequesser et al. ${ }^{43}$

For instance, the identity of NP-like content visualized in the endosomes of $C$. elegans treated with $6 \mathrm{~nm} \mathrm{Fe}_{2} \mathrm{O}_{3}$-NPs by TEM was confirmed by high angle annular dark field (HAADF) scanning transmission electron microscopy (STEM) coupled to EDX and also by EELS, revealing cell uptake of iron oxide particles by endocytosis (Fig. 10). Under HAADF STEM imaging modality, the intensity of the material is proportional to the square of the atomic number, $Z^{2}$. Hence, SPIONs appear with a higher intensity (that is, brighter) than the cellular background due to the higher atomic value of $\mathrm{Fe}(Z=26)$ compared to $\mathrm{C}(Z=6)$. EDX spectroscopy allows elemental identification by measuring the number and energy of X-rays emitted from a specimen after excitation with an electron beam. Conversely, EELS measures the energy loss when the sample is irradiated with an electron beam to determine the elemental components of the material.

Evaluation of the spectral properties of Au-NPs inside C. elegans, obtained by absorbance $\mu$-spectroscopy with $10 \mu \mathrm{m}$ precision, revealed a reversible aggregation pattern depending on the physiological features of the anatomical area investigated based on the peak position of the absorption maxima within the animal, which is related to NP size and degree of aggregation. ${ }^{64}$ Despite the fact that aggregation occurred, the position of the maximum absorption peak confirms that Au-NPs remain nano-size materials inside $C$. elegans. Merging the spectral information with the biodistribution results obtained by twophoton luminescent microscopy (TPLM), we could depict the findings regarding in vivo NP location and status on a single image, and evaluate the effect of NP size (Fig. 11). When illuminated with near-infrared pulsed light, Au-NPs luminesce via interband transitions induced by the absorption of two photons. Two-photon absorption is strongly enhanced when the pulsed illumination spectrally overlap with the localized plasmon resonance of the Au-NPs. ${ }^{79-83}$ Compared to bright-field or darkfield microscopy, TPLM offers enhanced contrast, is intrinsically 
A wild-type head region

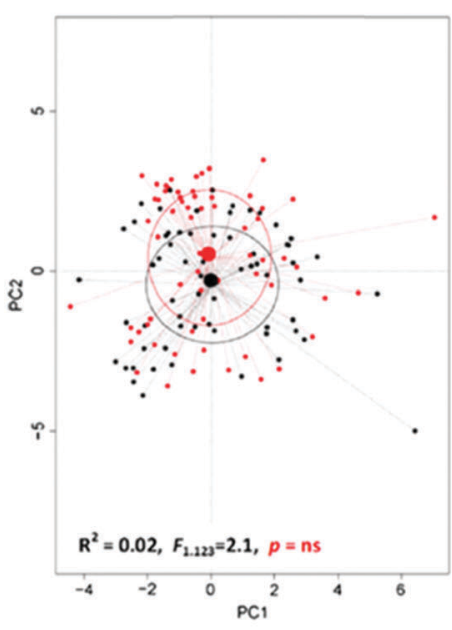

B

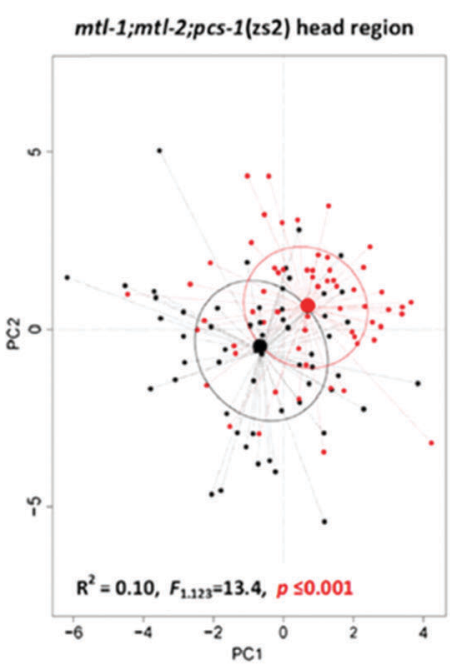

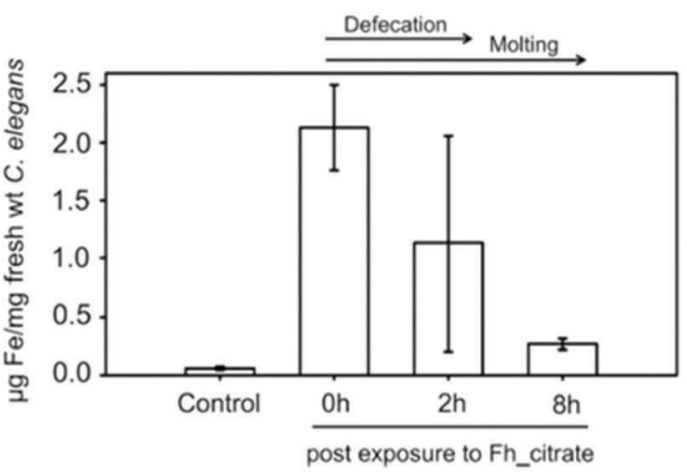

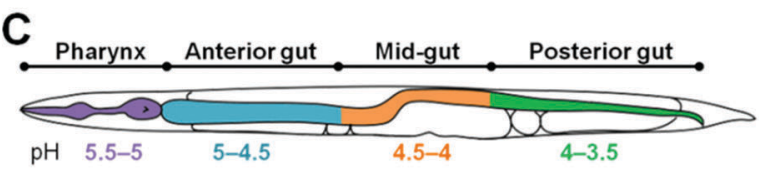

Fig. 9 Evaluation of NP uptake by analytical chemistry techniques. (A) Raman phenotype of ZnO-NP exposed and control animals (wild-type and $m$ tl$1 ; m t l-2 ; p c s-1$ mutants) in the head region. Principal component analysis shows the relationship between the Raman phenotype depending on the genetic background. Black points represent spectra from control nematodes, red points represent spectra from ZnO-NP exposed nematodes. Black and red ellipses represent standard deviations of point scores for the control and treatment groups, respectively. Adapted from Polak et al. ${ }^{49}$ (B) Fe concentrations measured in C. elegans after $6 \mathrm{~h}$ exposure to $\mathrm{K}$-medium (control) and ferrihydrite colloids associated with citrate (Fh_citrate) (28 mg Fe per L); $0 \mathrm{~h}$ post exposure: comprises bioaccumulated, attached and ingested Fe; $2 \mathrm{~h}$ post exposure: comprises bioaccumulated and attached Fe; $8 \mathrm{~h}$ post exposure: comprises bioaccumulated Fe; bars: arithmetic mean, error bars: standard deviation $(n=3)$. Adapted from Höss et al. ${ }^{27}$ (C) $\mathrm{pH}$ values of the intestinal tract of $C$. elegans, ranging from 5.5 in the pharynx to 3.5 in the posterior gut. Data extracted from Chauhan et al. ${ }^{10}$

A TEM

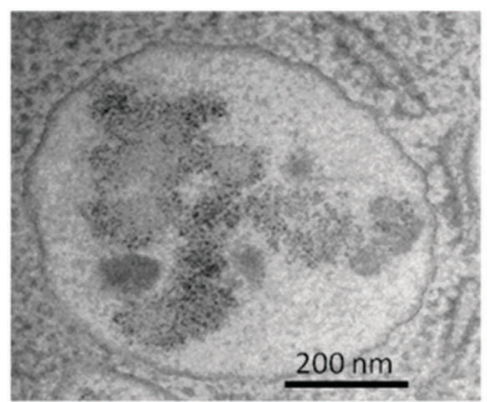

B HAADF STEM - EDX

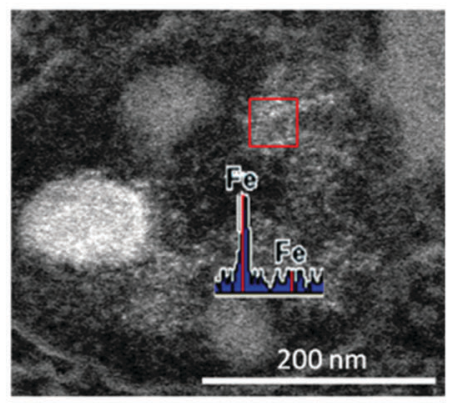

C EELS

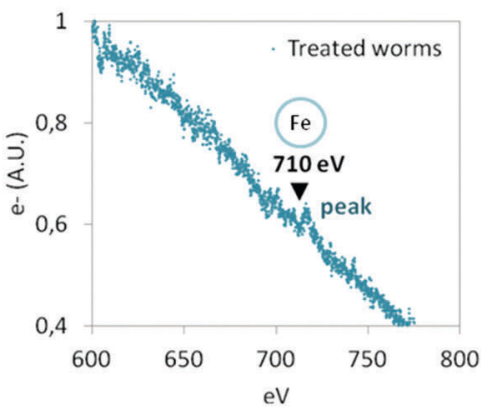

Fig. 10 Investigation of endocytosis of $6 \mathrm{~nm}$ iron oxide nanoparticles by the intestinal cells of $C$. elegans, combining the imaging and analytical capabilities of (A) TEM, (B) HAADF STEM coupled with EDX, and (C) EELS. The combination of these techniques allows the researcher to locate and identify NPs intracellularly in endosomes unambiguously. Adapted from Yu et al. ${ }^{68}$

confocal and offers three-dimensionality with increased spatial resolution. Remarkably, the combination of TPLM and absorbance $\mu$-spectroscopy allows depiction of both Au-NP biodistribution and aggregation status inside $C$. elegans in a single image, still providing a thorough material characterization.

Rare earth-doped upconverting nanoparticles such as lanthanide co-doped $\beta-\mathrm{NaYF}_{4}$ nanocrystals show promising applications for imaging in Caenorhabditis elegans, allowing a comprehensive study of their fate using infrared-based techniques. ${ }^{11,84}$ Indeed, these materials show the highest brightness near infrared to visible light up-converters, hence they are extremely interesting to investigate nanoparticles biodistribution and fate in C. elegans as an alternative to other fluorescent nanoparticles such as quantum dots.
3.7.2. Magnetometry to characterize ingested magnetic nanoparticles. Superconducting quantum interference devices (SQUID) are the most sensitive magnetic flux detectors and allow the measurement of very low magnetic moments. SQUID have been applied to determine the uptake of magnetic NPs by cells in vitro and we recently extended this approach to quantify the ingestion of magnetic NPs by C. elegans. ${ }^{34,85}$ Studying the magnetic hysteresis loops at low temperature (Fig. 12A and B) of magnetic NPs (i.e. superparamagnetic iron oxide nanoparticles, hereinafter SPIONs) and of treated worms, it is possible to determine the remanent magnetization, $M_{\mathrm{R}}$, that is, the magnetization value of a sample at zero applied field after the sample was fully magnetized. The $M_{\mathrm{RNPs}}$ and $M_{\mathrm{R} \text { worms }}$ derived from the ferrimagnetic behavior of the two samples are not affected by any diamagnetic signals, i.e. 

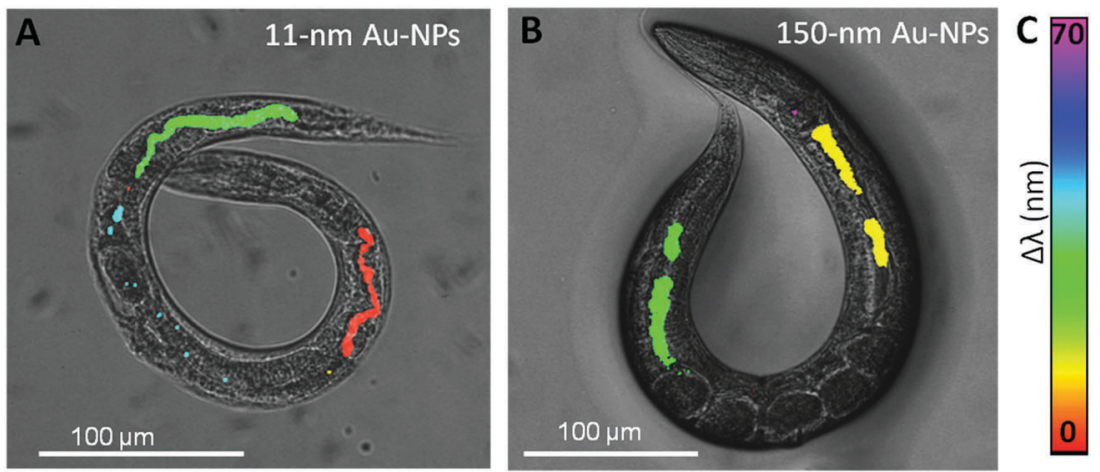

Fig. 11 Combination of two-photon luminescence microscopy and absorbance micro-spectroscopy to characterize nematodes treated with gold nanoparticles of (A) $11 \mathrm{~nm}$ Au-NPs and (B) $150 \mathrm{~nm}$. The two-photon luminescent signal from Au-NPs is merged with a dark-field micrograph of the treated animals, and colored according to the peak shift of the absorption maxima (by absorbance micro-spectroscopy) compared to the respective AuNP in dispersion. (C) Color legend of the peak shift (expressed in nanometers). Adapted from Gonzalez-Moragas et al. ${ }^{64}$

diamagnetism of the C. elegans tissue. Thus the ratio of $M_{\mathrm{RNPs}}$ and $M_{\mathrm{R} \text { worms }}$ will be informative regarding the NP uptake by $C$. elegans, providing the number of worms present in the sample is known. NP quantification by magnetometry has been contrasted with ICP-MS results with good agreement both in cells and C. elegans, confirming the value of this technique in the determination of the uptake of magnetic NPs in biological systems of increasing complexity. ${ }^{34,68,85}$ The quantitative data obtained in the magnetometry studies can be complemented with light microscopy studies of Prussian bluestained specimens, which provide a qualitative evaluation of NP biodistribution both in cells and C. elegans (Fig. 12C and D).

Magnetometry can also be applied to investigate NP fate by monitoring how the magnetic moment of the sample changes over temperature at a determined applied magnetic field. This measurement, known as Zero Field Cooled Field Cooled (ZFCFC), provides a characteristic parameter, the blocking temperature $\left(T_{\mathrm{B}}\right)$, that is related to properties of the NPs under study and is proportional to their volume. Comparing the initial $T_{\mathrm{B}}$ of magnetic NPs with the $T_{\mathrm{B}}$ of NP-treated C. elegans, it is possible to confirm the superparamagnetic properties of SPIONs in vivo and to determine their degradation profile. These results provide indirect evidence of NP size evolution, and have been successfully corroborated with much more laborious analysis of C. elegans cross-sections by TEM (Fig. $12 \mathrm{~F}$ and G). ${ }^{34,68}$ Both ZFC-FC measurements and TEM visualization supports the notion that NPs remain individual inside $C$. elegans. However under different conditions, we could hypothesize that some nanoparticles might aggregate.

To conclude this section, it is worth noting that when investigating the interaction between NPs and C. elegans, the transparency and small size of the worm facilitates the study of NP biodistribution under optical microscopy, enabling the visualization of its internal organs without need of dissection. ${ }^{2,86}$ More advanced imaging techniques including TEM, SEM, MRI, hyperspectral dark field microscopy (HDFM) or $\mu$-SRXRF can help to identify, locate and characterize individual NPs in specific regions of the body, and to unravel the mechanisms by which NP cross biological barriers in complex organisms. Together with spectroscopy techniques, they provide insights on the in vivo fate, aggregation and degradation of NPs, and allow the study of bio-accumulation and bio-persistence. Quantitative techniques like inductively coupled plasma mass spectrometry (ICP-MS) allow the quantification of NPs inside the worm to investigate dose- and time-dependence accumulation, and NP metabolism. Interestingly, some techniques not widely employed to date such as light sheet microscopy, two-photon luminescence microscopy, magnetometry or synchrotron techniques, allow the simultaneous characterization and quantitation of NPs inside treated animals. However, protocols for sample preparation might require specific adaptations for the C. elegans specimens. ${ }^{75,87}$ Table 2 summarizes the main characteristics of a variety of NP characterization techniques that have been, or can be, applied to evaluate inorganic NPs in C. elegans. The information they can yield, together with their main advantages and drawbacks, are also presented. The correlation of the physicochemical properties of the NPs with their in vivo status and toxicity can provide feedback to further optimize NPs within the synthetic laboratory to ensure maximum quality, efficiency and safety 'by design', a growing trend in the pharmaceutical industry at the early stages of discovery. ${ }^{88}$

\section{Biological responses triggered by metal and metal oxide NPs: approaches and common outcomes}

In the evaluation of metal and metal oxide NPs, it is important to note that some metals, known as essential metals, have a biological role in animals and plants. Zinc, copper, manganese and iron are essential metals for both humans and C. elegans, and they play an important role in diverse biological processes (Table 3). ${ }^{91,92}$ The same is true of the metalloid selenium. ${ }^{93,94}$ All of these, then, have a biphasic dose-response, in which either too little or too much is toxic. Metal homeostasis and transport of these essential elements have evolved over millennia and involve sophisticated mechanisms to regulate uptake and distribution within an organism, because an imbalance caused by either deficiency or overload can cause severe dysfunctions. ${ }^{91,95}$ In some cases, nonessential metals can be 
A
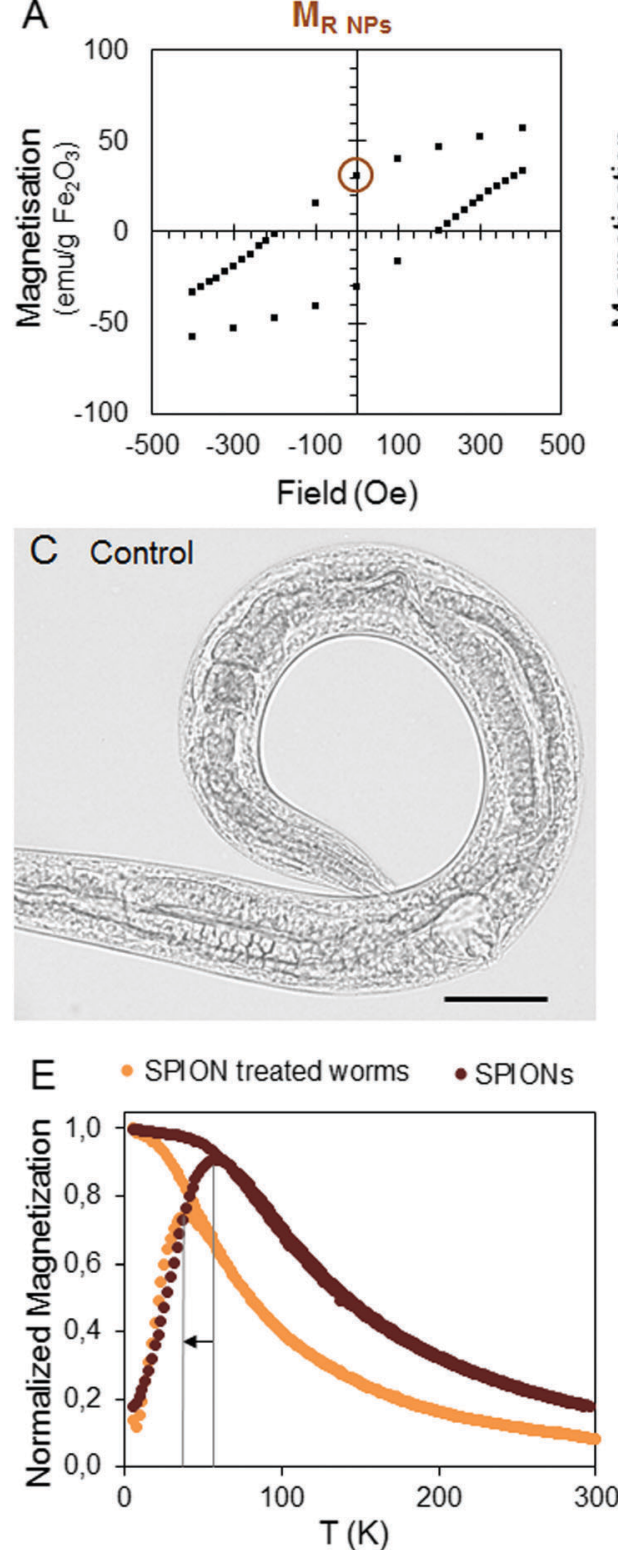

B

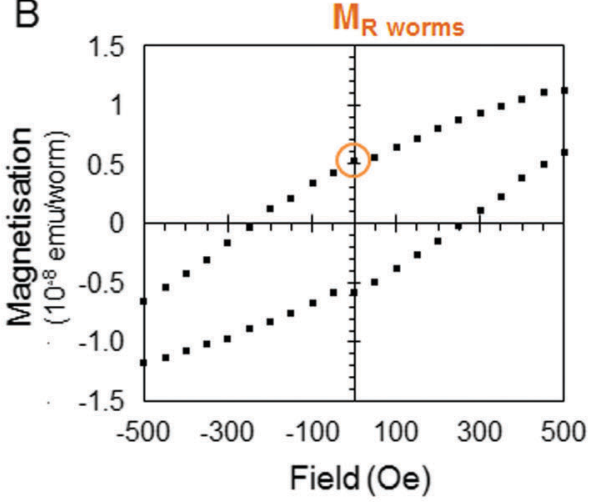

Treated C. elegans
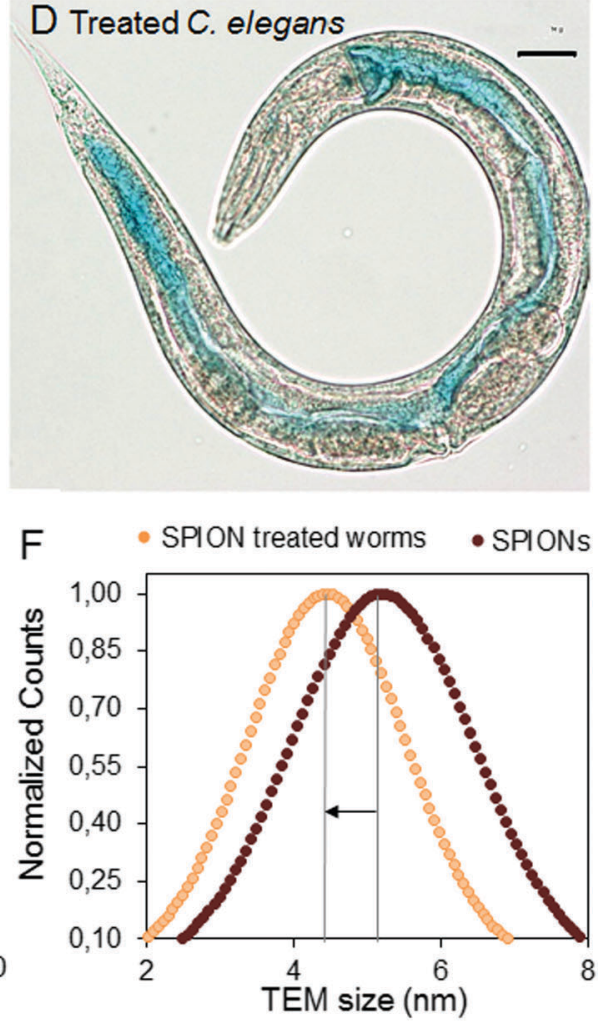

Fig. 12 Application of SQUID magnetometry and Prussian blue staining to the study of SPION-treated C. elegans. (A and B) Data processing of the magnetic characterization of animals treated with iron oxide NPs to quantitatively determine iron uptake. (A) Blow up of the magnetic hysteresis of $\mathrm{Fe}_{2} \mathrm{O}_{3}-\mathrm{NPs}$ at $5 \mathrm{~K}$ showing the remanence magnetization $M_{\mathrm{RNPs}}$. (B) Blow up of the magnetic hysteresis of treated worms at $5 \mathrm{~K}$ showing the remanence magnetization $M_{R \text { worms. }}$ ( $D$ and E) Prussian Blue staining facilitates NP visualization and confirms chemical composition. (D) Optical microscopy image of a control C. elegans. Scale bar $=30 \mu \mathrm{m}$. (E) Optical microscopy image of a SPION-treated C. elegans stained with Prussian blue. Scale bar $=30 \mu \mathrm{m}$. (F and G) Investigation of SPION size inside treated C. elegans by SQUID magnetometry and TEM. (F) ZFC-FC plots of SPIONs and SPION-treated C. elegans. By comparing the $T_{\mathrm{B}}$ values, it is possible to study the biotransformation of SPIONs inside $C$. elegans. (G) TEM size distribution analysis in cross-sections of treated C. elegans confirm the good agreement with magnetometry. ${ }^{34,68}$

taken up and transported throughout the body by mimicry of essential metals; for example, $\mathrm{Ag}$ can mimic $\mathrm{Cu}, \mathrm{Cd}$ can mimic $\mathrm{Zn}, \mathrm{Pb}$ can mimic $\mathrm{Ca}$, etc. C. elegans has been used as a model organism in the study of metals in biology and has shown that accumulation of metals depends on the physicochemical properties of metals, the physiology of organisms and the nature of the metal (essential or not). ${ }^{91,92,95-97}$ This work comprises an excellent body of literature on which to draw when studying the biological effects of metals-based NPs.
In addition, it is also important to consider that some NPs can release metal ions in the exposure media or inside the organism, adding their effects to the nano-specific effects. Evaluation of ion leakage from the NP core has been investigated typically at the lysosomal $\mathrm{pH}$, mimicking intracellular conditions; the kinetics of degradation depend on NP composition, size and coating. Fig. 13 summarizes the different dissolution rates for different NP compositions at $\mathrm{pH} \sim 4.5$. 


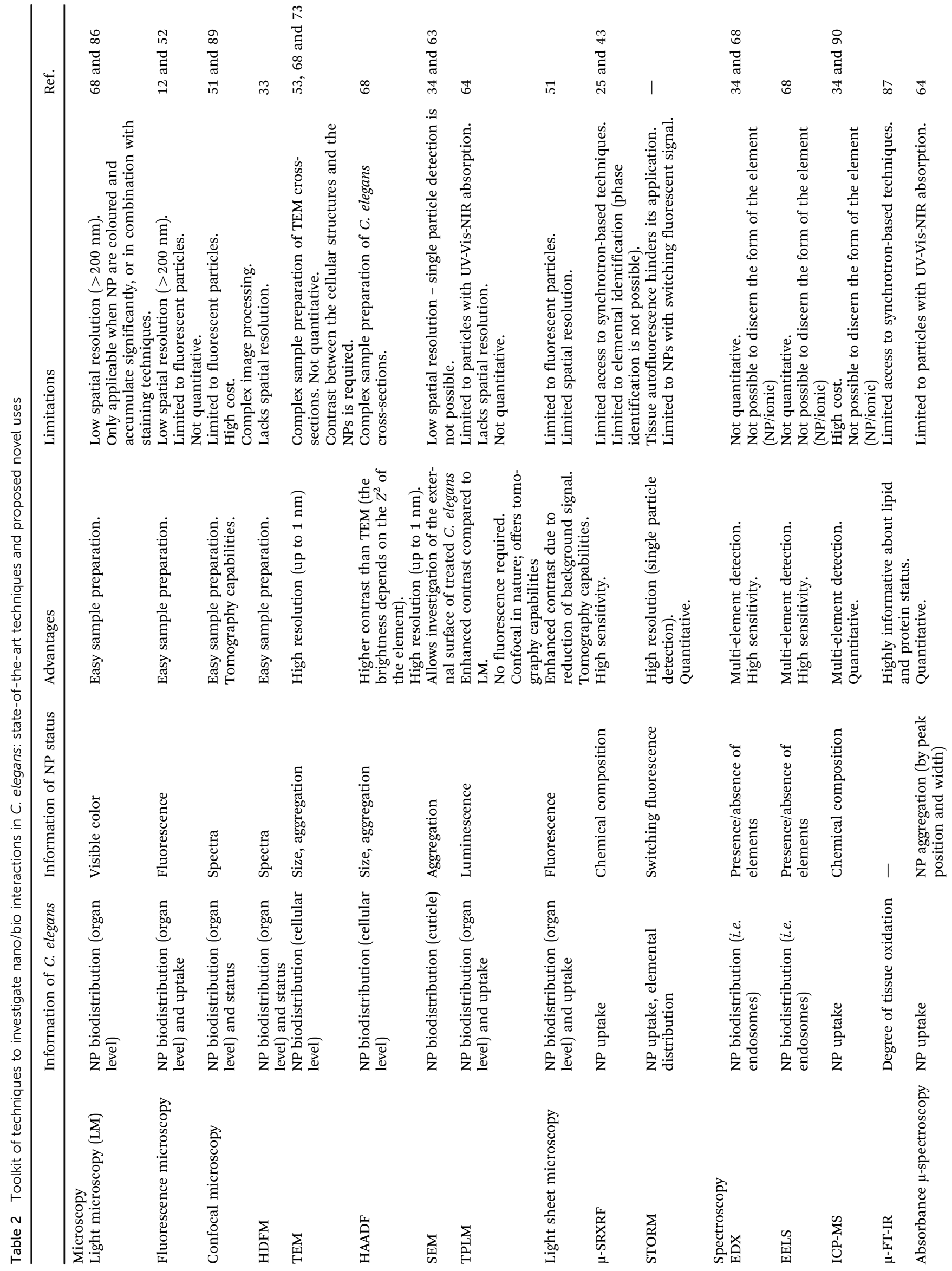


Different strategies have been applied to discern the molecular mechanisms of toxicity triggered by NP exposure in C. elegans. These include assays of production of reactive oxygen species (ROS) production or damage to macromolecules (e.g., biochemical assays to detect ROS formation, measures of DNA damage, imaging protocols to quantify lipofuscin accumulation); assays of biological response that may be indicative of engagement of a damage response pathway (e.g., studies of altered gene or protein expression, including transcriptomics); "rescue" experiments (e.g., pharmacological co-exposures, such as with antioxidant treatment); or genetic approaches to "functional toxicology", such as the use of mutant strains or RNAi. ${ }^{38,49,54,104}$

Each of these approaches has strengths and limitations. Damage to macromolecules has the strength of clear biological relevance; however, it is not always possible to distinguish whether the damage is a direct or indirect effect of exposure. In addition, it is uncommon to measure multiple types of damage in the same study, making it difficult to determine which targets are the most sensitive. Finally, these assays tend to be less sensitive than others, because such damage is typically repaired as long as homeostasis is maintained, such that stress may not be reflected as damage until defenses are overwhelmed. Altered regulation of gene or protein expression may be informative of NP mechanism of toxicity; for example, upregulated antioxidant genes may reflect that the NP caused oxidative stress, triggering an adaptive or defensive response via regulation of appropriate stress-response pathways. Table 4 summarizes frequently investigated genes and pathways in NP toxicity. Limitations associated with these approaches include the fact that many stress-response genes are regulated by multiple response elements, and may therefore not be specific with respect to the mechanism of action of the stressor. Assessment of multiple gene/protein components of multiple stress response pathways (Table 5) helps with interpretation. Pharmacological rescue experiments (e.g., if co-exposure with an antioxidant compound rescues/reduces toxicity, this would suggest that oxidative stress is a mechanism of toxicity), or their converse, are powerful to the extent that they directly modulate (exacerbate or protect against) a specific mechanism of toxicity. However, in some cases these assays may be hindered by lack of specificity or by difficulty in targeting the compound to the site of action.

Finally, genetic approaches are relatively less used by the toxicological community than the approaches listed above. Therefore, we describe them in more detail and exemplify their use in nanotoxicological research. Genetic approaches involve manipulating the genome of an organism to probe how this alteration affects the biological response to a stressor. For example, if a metal nanoparticle causes toxicity by dissolving and releasing a toxic metal cation, genetic mutation or deletion of the gene metallothionein ( $m t l-1$ and $m t l-2$ in C. elegans), which encodes a polypeptide that chelates metal ions and thus protects against their toxicity, should exacerbate the toxicity of that NP. In this line, selected strains of C. elegans lacking mitochondrial homeostasis genes were exposed to multiple doses of positively charged amine-coated $9 \mathrm{~nm}$ Ag-NPs (Fig. 14). The eat-3 
Table 3 Biology of select metals in C. elegans. Data extracted from Chen et al. ${ }^{91}$ and Li et al. ${ }^{93}$

\begin{tabular}{|c|c|c|c|c|c|}
\hline & Copper & Iron & Manganese & Zinc & Selenium \\
\hline Functions & $\begin{array}{l}\text { - Iron homeostasis } \\
\text { - Neurotransmitter bio- } \\
\text { synthesis } \\
\text { - Oxidative phosphory- } \\
\text { lation, } \\
\text { - Oxidative stress } \\
\text { protection }\end{array}$ & $\begin{array}{l}\text { - DNA synthesis } \\
\text { - Mitochondrial respiration } \\
\text { - Oxygen transport } \\
\text { - Neurotransmitter synthesis. }\end{array}$ & $\begin{array}{l}\text { - Fat and carbohydrate } \\
\text { metabolism } \\
\text { - Oxidative stress } \\
\text { protection (SOD) } \\
\text { - Neurotransmitter synthesis } \\
\text { and metabolism. }\end{array}$ & $\begin{array}{l}\text { Cofactor in several cel- } \\
\text { lular processes and cel- } \\
\text { lular signaling } \\
\text { pathways. }\end{array}$ & $\begin{array}{l}\text { - Development } \\
\text { - Reproduction } \\
\text { - Antioxidant } \\
\text { activity } \\
\text { - Neuroprotection }\end{array}$ \\
\hline Deleterious & Deficiency & Deficiency & Excess & Excess & Excess \\
\hline effects & $\begin{array}{l}\text { Decreased SOD, redu- } \\
\text { cing defenses against } \\
\text { oxidative stress. } \\
\text { Excess } \\
\text { Detrimental effects on } \\
\text { brood size and life span, } \\
\text { an increase in genera- } \\
\text { tion time and impaired } \\
\text { development. }\end{array}$ & $\begin{array}{l}\text { HIF-1 inhibits } f t n-1 \text { and } f t n-2 \\
\text { transcription. The activation of } \\
\text { smf-3 provides a mechanism to } \\
\text { maintain sufficient Fe stock for } \\
\text { growth and survival. } \\
\text { Excess } \\
\text { Phenotypic and behavioural } \\
\text { defects like reduced lifespan, } \\
\text { brood size, locomotion, and } \\
\text { alters the resistance to } \\
\text { oxidative stress }\end{array}$ & $\begin{array}{l}\text { Accelerated development, } \\
\text { increase in fertility, reduced } \\
\text { body and brood size and } \\
\text { life span. } \\
\text { Increased ROS formation and } \\
\text { glutathione production, head } \\
\text { mitochondria membrane } \\
\text { potential and dopaminergic } \\
\text { neuronal death. }\end{array}$ & $\begin{array}{l}\text { Multiple biological } \\
\text { defects affecting life } \\
\text { span, reproduction, } \\
\text { locomotion behaviour } \\
\text { and chemotaxis } \\
\text { plasticity. }\end{array}$ & $\begin{array}{l}\text { Decreased devel- } \\
\text { opmental rate and } \\
\text { brood size; } \\
\text { Neurotoxicity; Oxi- } \\
\text { dative stress. }\end{array}$ \\
\hline Proteins & \multicolumn{4}{|c|}{$\begin{array}{l}\text { Highly conserved: } \mathrm{Cu} / \mathrm{Zn} \text { superoxide dismutase (SOD), divalent metal transporters (SMF-1, SMF-2, SMF-3), Ca }{ }^{2+} / \\
\mathrm{Mn}^{2+} \text { ATPas (PMR1), homolog of mammalian Nrf2 (SKN-1), cation diffusion facilitators (CDFs), Zrt- and Irt-like } \\
\text { proteins (ZIPs), ferritin (FTN-1, FTN-2), Fe sulfuric cluster assembly proteins, metallothioneins (MTs). }\end{array}$} & $\begin{array}{l}\text { Glutathione per- } \\
\text { oxidases (GST), } \\
\text { thioredoxin reduc- } \\
\text { tase (TRXR-1) }\end{array}$ \\
\hline
\end{tabular}

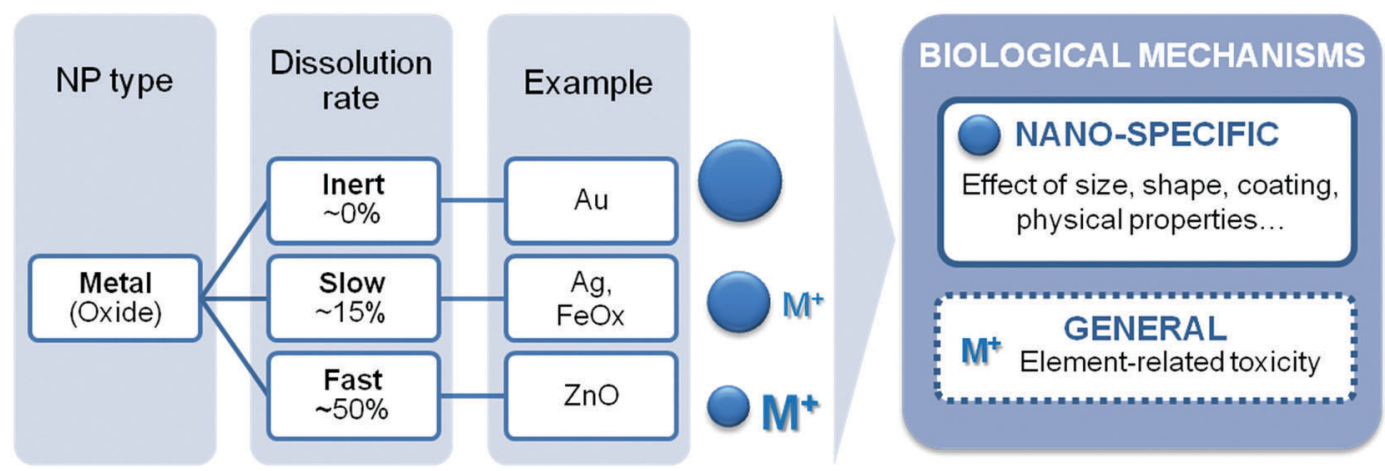

Fig. 13 Biological mechanisms of metal and metal oxide nanoparticles. The left panel exemplifies the dissolution rates of different NP types based on a time frame of 24-48 h in biologically relevant media. The right panel shows the different mechanisms of action expected for inorganic NPs in vivo. These are a combination of nano-specific effects and, in the case of biodegradable particles, also ion-related effects. Data extracted from Ma et al., Liu et al., ${ }^{98}$ Luo et al., ${ }^{99}$ Yu et al., ${ }^{68}$ Levy et al.., ${ }^{100}$ Soenen et al., ${ }^{101,102}$ Sabella et al., ${ }^{103}$ Ma et al. ${ }^{24}$ and Polak et al. ${ }^{49}$

strain, which is deficient in mitochondrial fusion, was more sensitive than the others, suggesting that mitochondrial fusion is important in protecting against the toxicity of these NPs, and that mitochondria are a target of their toxic effects. Genetic manipulations may be achieved by mutation/deletion of genes resulting in mutant (e.g., knockout) or known down strains, by reducing the level of protein produced using methods such as RNA interference (RNAi), or by overexpressing specific genes and proteins. Such genetic approaches were recently dubbed "functional toxicology" in the context of toxicology, ${ }^{104}$ and their results are particularly convincing when the function of the protein involved is fully understood and highly specific. However, this is not always the case; for example, while metallothionein knockdown appears to result in a fairly specific form of stressor sensitivity (metal ions), knockdown of some genes may have multiple impacts, complicating interpretation. For example, knockdown of superoxide dismutase (sod genes) reduces the ability to detoxify superoxide anion, but also reduces the ability to produce hydrogen peroxide, which has important signaling functions.

The effects and responses observed in the screening of metal and metal oxide NPs in C. elegans are summarized in Fig. 15. Overall, as with materials science approaches, the most compelling results are typically those obtained combining different methods, each complementing the others by compensating for the intrinsic limitations that each has.

Table 5 presents different biological mechanisms reported to be triggered by NP exposure, illustrating discrepancies among the conclusions of different studies. For instance, some studies using Ag-NPs concluded that oxidative stress was an important factor determining Ag-NP toxicity, ${ }^{38,59,63}$ whereas other authors were not able to find a link between Ag-NP toxicity and oxidative stress, ${ }^{33}$ and others proposed that NPspecific effects were restricted to the less soluble due to size or coating. ${ }^{36}$ Similarly, we found controversial results concerning 
Table 4 Frequently investigated genes and pathways in NP toxicity

Pathway

General stress

Metal stress

Oxidative stress

DNA damage

Metabolic stress

Mitochondrial function

Collagen

Endocytosis

Lysosomal function

Non-canonical UPR

ER stress

Metabolism

Yolk proteins

MAPK signalling pathway
C. elegans gene

hsp-16.2, hsp-16.41, hsp-16.48, daf-2, daf-12, daf-16, daf-21, sgk-1, akt-1, akt-2. $m t l-2, p c s-1, m t l-1, m t l-2, c d r-1, p c s-1$

sod-1, sod-2, sod-3, sod-4, sod-5, ctl-1, ctl-2, ctl-3, mev-1, nth-1

cep-1, ced-3, ced-4, xpa-1, nth-1, ape-1

ire-1, sir-2, aak-2

gas-1, coq-7

col-158, col-131, col-101

rme-1, rme-6, rme-8, dyn-1, chc-1, rme-2

cup-5, glo-1

abu-11, pqn-5, hsp-16.1, hsp-70, hsp-3, hsp-4

hsp-4

gst-1, gst-4, gst-5, gst-8, gst-24, and gst-42, age-1, gas-1, cyp35a2

vit-2, vit-6

jnk-1, mpk-2, nsy-1, sek-1, pmk-1, jkk-1

Table 5 Biological mechanisms triggered by NP exposure reported in the selected literature

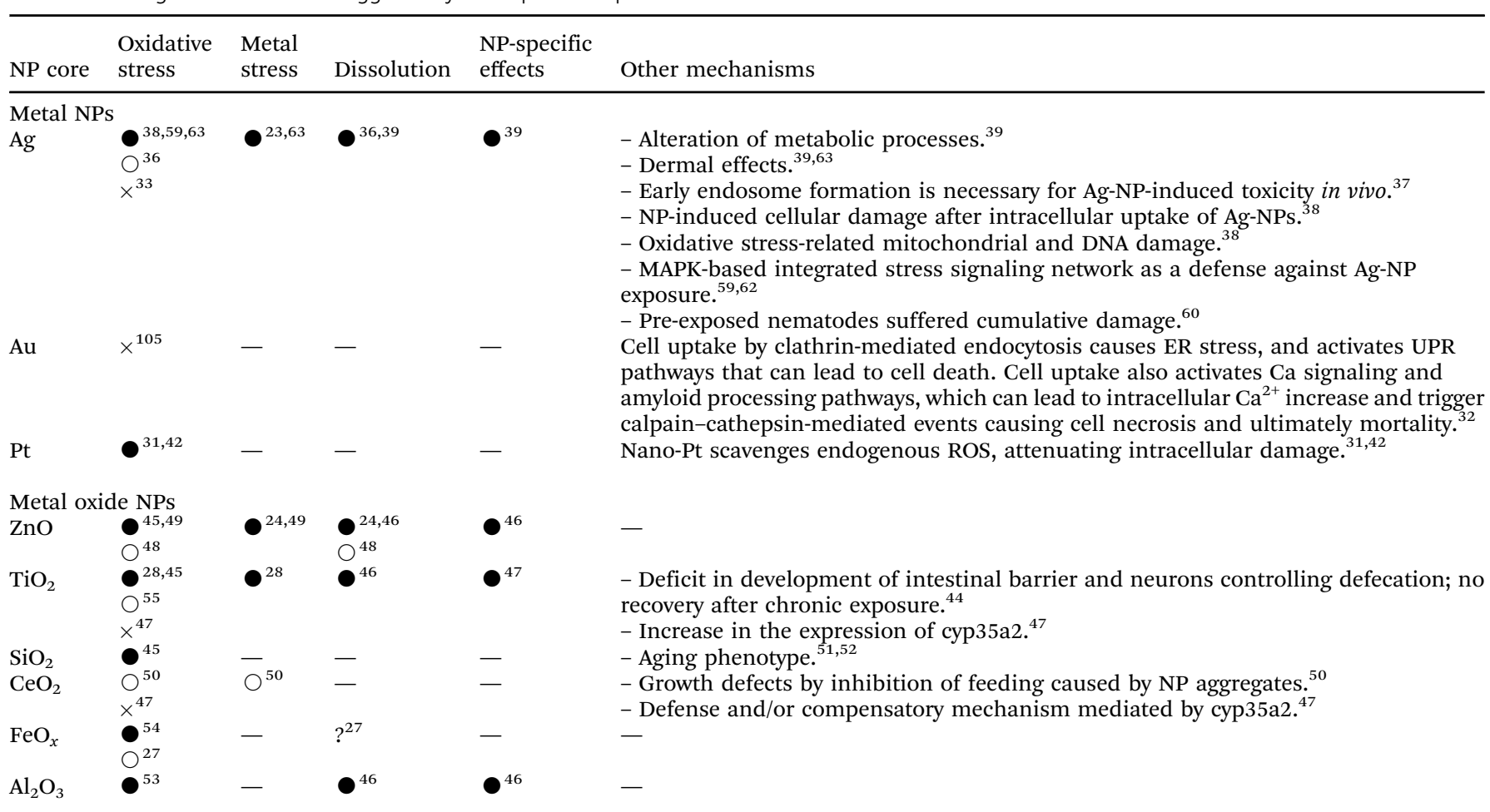

Major role, $\bigcirc$ minor role, $\times$ lack of evidence, hypothesis.

iron oxide NPs: some studies reported a correlation between oxidative stress and NP toxicity (either major ${ }^{54}$ or minor $^{27}$ ) and also proposed that dissolution inside C. elegans and ion release could occur, contributing to the biological effects on vivo. ${ }^{27,67}$ Therefore, to date there is no clear evidence supporting a single, general biological mechanism triggered by NP exposure, nor for specific mechanisms that are predictable depending on NP properties such as composition, size or coating.

\subsection{Pathways by which metal and metal oxide NPs cross biological barriers}

Given the technical difficulty of studying NP fate with nanometric resolution inside living organisms at present, there is limited evidence of the internalization and translocation mechanisms of NPs in C. elegans. In vitro assays have shown that upon internalization, the surface functionality of NPs dictates their behavior and subcellular location. ${ }^{106}$ It is worth noting that different cellular compartments have different characteristics (i.e. the cytoplasm and lysosomes have different redox status and $\mathrm{pH}$ ); hence, the local environment of NPs may influence their reactivity and oxidation status. Moreover, the negative membrane potential of most cells interacts differently with particles with a positive or negative surface charge. The electrostatic interaction of NPs with the negatively charged bilayer of a membrane mediates their binding and their toxicity. Therefore, many aspects at the cellular scale deserve to be further addressed in vivo to better understand the complex nano/bio interactions occurring inside living organisms. 

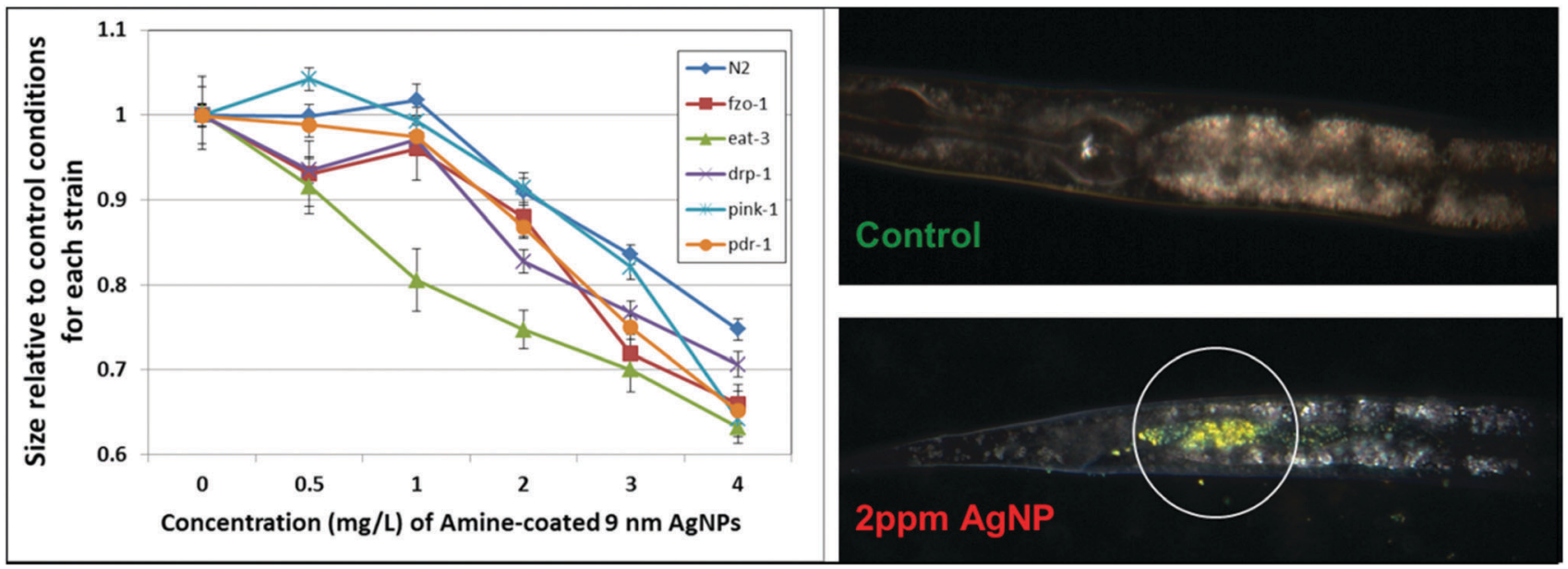

Fig. 14 Use of a genetic approach to test a mitochondrial mechanism of toxicity of AgNPs. We tested the sensitivity of N2 and multiple mutant strains with deficiencies in mitochondrial homeostasis genes (drp-1, eat-3, fzo-1, pdr-1, pink-1) to exposure to 9 nm (average diameter), amine-coated, positively-charged AgNPs. The graph depicts the relative length of control and treated animals after $72 \mathrm{~h}$ of growth from the L1 stage (synchronized), in MHRW, with UVC-killed UVRA bacteria. Error bars represent one standard error of the mean; experiment repeated twice in time, total $n=39-40$ nematodes per dose per strain. Because aggregation of AgNPs was observed, we used hyperspectral imaging to verify ingestion after $48 \mathrm{~h}$ with a $2 \mathrm{ppm}$ exposure. Ingestion was observed for all strains (N2 shown). Unpublished data from Victoria Harms, Laura Maurer, and Joel Meyer; AgNPs synthesized by Stella Marinakos; Cytoviva images taken and analyzed by Nick Geitner.

\section{ORGANISMLEVEL}

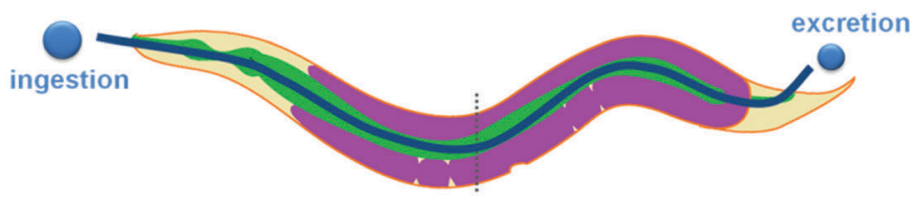

biological effects and biotransformation

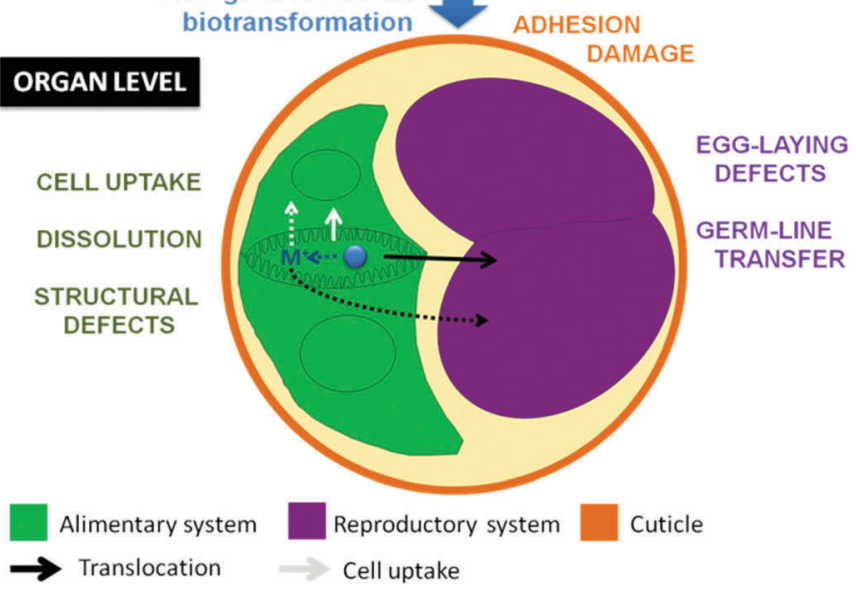

\section{CELLULAR AND SUBCELLULAR LEVEL}
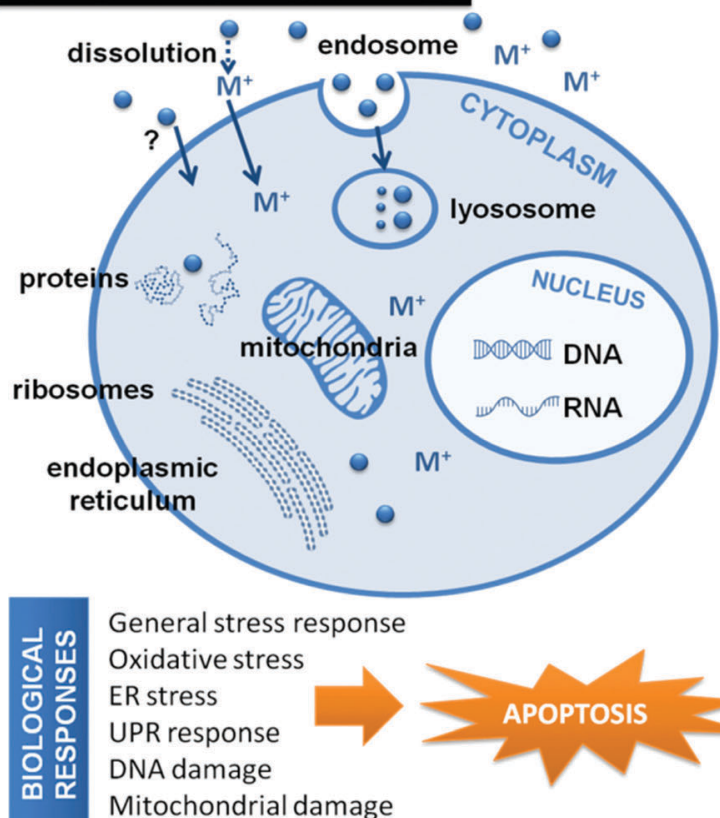

General stress response

Oxidative stress

ER stress

UPR response

DNA damage

Mitochondrial damage

Fig. 15 Effects and mechanisms observed in the screening of metal and metal oxide NPs in C. elegans at the organism, organ, cellular and subcellular levels. At the organism level, NP treatment has been found to affect several toxicity endpoints including survival, growth, brood size, locomotion and pharyngeal pumping, among others. At the organ level, egg-laying defects and NP intergenerational transfer have been reported. Adverse effects on the dermal and intestinal barrier have also been identified after chronic treatment. For biodegradable particles such as $\mathrm{ZnO}_{\mathrm{n}}$ or $\mathrm{Fe}_{2} \mathrm{O}_{3}, \mathrm{NP}$ metabolism inside $\mathrm{C}$. elegans has been demonstrated using a range of techniques, including magnetometry or spectroscopy. Regarding the molecular mechanisms reported after $C$. elegans treatment with metal and metal oxide NPs, different stress pathways have been identified including general stress, oxidative stress or endoplasmic reticulum (ER) stress. These responses could activate signaling cascades (i.e. UPR, calcium, MAPK) leading to organellar effects (i.e. mitochondrial dysfunction, lysosomal impairment, DNA damage) and finally, result in premature cell death by apoptosis. UPR: unfolded protein response.

Meyer $e t$ al. reported the first direct evidence of intracellular uptake and intergenerational transfer of $10 \mathrm{~nm}$ citrate Ag-NP in C. elegans using hyperspectral microscopy, but did not perform mechanistic studies of how this uptake occurred. Using fluorescence microscopy, Scharf et al. also observed intracellular uptake of $50 \mathrm{~nm} \mathrm{SiO}_{2}$-NP in the intestinal and vulval cells. ${ }^{51}$ 
Employing transcriptional and genetic analysis tools, Tsyusko et al. reported elevation of $c h c-1$ expression and significant responses of endocytosis mutants (chc-1 and rme-2) to Au-NPs, suggesting that cell uptake of Au-NPs occurs via clathrinmediated endocytosis. ${ }^{32}$ Recently, Maurer et al. showed that early endosome formation is necessary for Ag-NP-induced toxicity in vivo using endocytosis-deficient mutants (rme-1, rme-6 and rme-8) and lysosomal function deficient mutants (cup-5 and glo-1), as well as a clathrin-mediated endocytosis inhibitor. ${ }^{37}$ Gonzalez-Moragas et al. reported intracellular

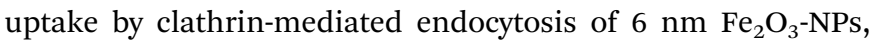
and down-regulation of the early endosome formation gene $d y n-1$, among other intestinal-related genes, however internalization of $11 \mathrm{~nm}$ Au-NPs were not detected either by electron microscopy or by gene expression analysis. ${ }^{68,74}$

\subsection{Toxicological mechanisms of metal nanoparticles}

4.2.1. Gold NPs as a model system. In an attempt to study particle-specific effects of manufactured nanomaterials, Tsyusko et al. chose gold NPs as a model since they are resistant to oxidative dissolution. As part of a larger-scale experiment investigating interactions between metals and NPs, Tsyusko et al. investigated the transcriptomic effect of exposure to $4 \mathrm{~nm}$ citrate Au-NPs for $12 \mathrm{~h}$ in L3 nematodes. ${ }^{32}$ The authors proposed that Au-NPs are taken inside the cell through clathrin-mediated endocytosis and once inside the cell, they cause endoplasmic reticulum (ER) stress, resulting in increased amounts of misfolded or unfolded proteins and activation of canonical (molecular chaperones) and noncanonical UPR pathways. Excess accumulation of misfolded proteins can lead to cell death. Furthermore, Au-NPs also seemed to activate $\mathrm{Ca}$ signaling and amyloid processing pathways, which would lead to intracellular $\mathrm{Ca}^{2+}$ increase and trigger calpaincathepsin-mediated events potentially leading to cell necrosis and ultimately to mortality. The results from this study provided evidence that $4 \mathrm{~nm}$ citrate Au-NPs can be internalized by the C. elegans cells and transcriptionally activate multiple biological pathways that respond to, or are capable of, causing adverse effects on whole organisms.

4.2.2. Toxicity mechanisms of Ag-NPs. Ellegaard-Jensen et al. hypothesized that $C$. elegans resistance to nano-silver would increase via physiological adaptation after pre-exposure to low concentrations. In contrast, they found that pre-exposed nematodes were slightly more sensitive to further exposure compared to non-pre-exposed animals. ${ }^{60}$ In good agreement, Contreras et al. showed that Ag-NP pre-exposed nematodes suffered cumulative damage. ${ }^{61}$ These results suggest that C. elegans has limited efficient physiological ability to counteract nano-silver toxicity by acclimation.

Roh et al. reported concentration-dependent, increased expression of mitochondrial superoxide dismutase (sod-3) and metallothionein ( $m t l-2)$ occurring concurrently with significant decreases in fertility. The authors interpreted this information to indicate that oxidative stress played an important mechanism in Ag-NPs toxicity. Meyer et al. also studied the roles of oxidative stress and metal genes, in this case using genetic (mutant analysis) techniques, and reported greater Ag-NP sensitivity of a $m t l-2$ deficient mutant, but did not find evidence for a role for oxidative stress in Ag-NP toxicity. The authors attributed at least part of the toxicity observed to dissolved silver. ${ }^{33}$ In contrast, Ellegaard-Jensen evaluated Ag-NPs of different size (1 and $28 \mathrm{~nm}$ ) and coating (bare or PVP) and found that toxicity was dependent on the type of Ag-NP but not on the soluble fraction alone. In a later study, Roh et al. reported the formation of ROS and analyzed the expression of genes in MAPK signaling pathways. They found an increase in gene expression of less than 2 -fold compared to control worms, but a dramatic increase in sod-3 mutants after exposure, suggesting a PMK-1 p38 MAPKdependent activation. The authors proposed that MAPK-based integrated stress signaling network could be involved in defense to Ag-NPs exposure in C. elegans. ${ }^{62}$ Studies by the same group confirmed increased ROS formation after Ag-NP exposure, as well as increased expression of PMK-1 p38 MAPK and hypoxiainducible factor (HIF-1), GST enzyme activity, and decreased reproductive potential in $C$. elegans. The authors found evidence that oxidative stress was an important mechanism of Ag-NPinduced reproduction toxicity in C. elegans, and that PMK-1 p38 MAPK played an important role in it. ${ }^{59}$

Yang et al. observed a linear correlation between Ag-NP toxicity and dissolved silver, and concluded that Ag-NP toxicity was driven at least in part by release of dissolved silver, in agreement with Meyer et al., even though oxidative dissolution was limited (maximally 15\%). Given that the bioavailability of ionic silver can be reduced by binding to glutathione (GSH) or thiol-containing proteins such as metallothionein, the authors showed that growth inhibition could be rescued by complexation of dissolved silver with thiol groups, while partial or no rescue was observed with antioxidants. This study highlighted a critical role for dissolved silver in the toxicity of all tested Ag-NPs, and also proposed a specific nano-Ag effect via oxidative stress typically for the less soluble Ag-NPs, hence encompassing the two most prevalent Ag-NPs toxicity mechanisms reported previously. ${ }^{36}$ Starnes et al. reported that the mechanism of AgNP toxicity was dependent on the concentration: at low concentrations, a greater proportion of the toxicity could be explained by dissolved $\mathrm{Ag}$, whereas at high concentration, the toxicity appeared to be dominated by particle specific effects. ${ }^{39}$ Recently, Ahn et al. combined qPCR studies, the use of mutants and biochemical assays to study the role of different genes related to general stress, immune response, metal stress, oxidative stress, metabolic stress and DNA damage in nematodes treated with AgNPs, and concluded that oxidative stress-related mitochondrial and DNA damage could be a potential mechanism of toxicity, in particular for the smaller Ag-NPs irrespective of their coating. ${ }^{38}$

Differences in the Ag-NP toxicity mechanisms and their relative importance reported in literature could arise from differences in the experimental design such as developmental stage or strain of $C$. elegans; exposure conditions including exposure media, food source, temperature, or exposure duration; but also from differences in the nanomaterial concentration, properties (size, coating) and colloidal stability. Overall, the data is consistent with multiple mechanisms of action playing larger and smaller roles in different contexts. 
4.2.3. Antioxidant properties of Nano-Pt. Kim et al. investigated the anti-ageing properties of Pt-NPs, which have superoxide dismutase (SOD)/catalase activity. Treatment of C. elegans with Pt-NPs significantly reduced the accumulation of lipofuscin and ROS induced by paraquat, conferring resistance against oxidative stress and prolonging lifespan by $25 \% .^{31}$ The authors proposed that in normal culture conditions, C. elegans maintain an excessive ROS level, hence the scavenging of endogenous ROS by nano-Pt attenuates intracellular damage and induces extension in lifespan. ${ }^{42}$

\subsection{Toxicological mechanisms of metal oxide nanoparticles}

4.3.1. The role of dissolution. Ma et al. investigated the toxicity of $\mathrm{ZnO}-\mathrm{NPs}$ and did not find differences from $\mathrm{Zn}^{2+}$ at the same molar $(\mathrm{Zn})$ concentrations. The two treatments induced expression of $m t l-2$ in a similar manner. These findings suggest biotransformation of the NPs to $\mathrm{Zn}^{2+}$ to enact toxicity, which was recently supported by spectroscopy by Savoly et al. ${ }^{24,107}$ Wang et al. also reported similar lethal doses $50\left(\mathrm{LC}_{50}\right)$ of $\mathrm{ZnO}-\mathrm{NPs}$ and bulk $\mathrm{ZnO}$, while $\mathrm{Al}_{2} \mathrm{O}_{3}$-NPs and $\mathrm{TiO}_{2}$-NPs were twice as toxic as their bulk counterparts. Hence, their toxicity could not be adequately explained by dissolution of the particles alone. ${ }^{46}$

4.3.2. Oxidative stress. $\mathrm{Wu}$ et al. observed a correlation between ROS production and toxicity in nematodes chronically exposed to environmentally relevant concentrations of $\mathrm{TiO}_{2}$ NPs, ZnO-NPs and $\mathrm{SiO}_{2}$-NPs, and showed that antioxidant treatment effectively rescued adverse effects. ${ }^{45}$ Rui et al. investigated the effects of $\mathrm{TiO}_{2}$-NPs in mutants associated with oxidative stress and stress response (among them, some metallothioneins, SOD, glutathione $S$-transferase, and heat shock protein mutants) and found that they were more susceptible when exposed to high dose for $24 \mathrm{~h}$, but not at lower doses or for shorter periods. ${ }^{28}$ Roh et al. exposed C. elegans to $\mathrm{CeO}_{2}-\mathrm{NPs}$ (15 and $45 \mathrm{~nm}$ ) and $\mathrm{TiO}_{2}(7$ and $20 \mathrm{~nm}$ ) and studied the expression levels of stress-responsive genes including metal response proteins, xenobiotic metabolism enzymes, antioxidant enzymes, apoptosis markers, and yolk proteins. Whereas the expression of most of the tested genes did not change significantly, likely due to the low dose used $\left(1 \mathrm{mg} \mathrm{L}^{-1}\right)$, the expression of cyp35a2 significantly increased after treatment. ${ }^{47}$ This result is surprising since cytochrome P450s (CYPs) are involved in the synthesis and metabolism of organic molecules, and do not have a known catalytic effect on inorganic particles. However, cyp35a2 is a highly stress-responsive gene, and these results may highlight the principle that induction of a gene does not prove that the mechanism of toxicity of the stressor is closely related to the biological function of the gene induced, since genes are often inducible by multiple stressors. Induction of different $C$. elegans CYPs after NP exposure was observed after treatment of C. elegans with $\mathrm{Fe}_{2} \mathrm{O}_{3^{-}}$, $\mathrm{TiO}_{2^{-}}$and $\mathrm{CeO}_{2}$-NPs. ${ }^{47,74,108}$

$\mathrm{Li}$ et al. studied chronic exposure of C. elegans to 0.01-23.1 $\mathrm{mg} \mathrm{L}^{-1} 60 \mathrm{~nm} \mathrm{Al}{ }_{2} \mathrm{O}_{3}$-NPs and reported a severe stress response including oxidative stress. These effects were much more subtle in bulk $\mathrm{Al}_{2} \mathrm{O}_{3}$, and not observed in acute exposure regimes. Oxidative stress was caused by both an increase in
ROS production and suppression of ROS defense mechanisms. Treatment with antioxidants and SOD-3 overexpression suppressed oxidative stress and prevented NP adverse effects, while sod-2 and sod-3 (both mitochondrial SODs) mutants were more susceptible than the wild-type to NP treatment. ${ }^{53}$ Exposure of C. elegans to DMSA-coated $\mathrm{Fe}_{2} \mathrm{O}_{3}$-NPs $(9 \mathrm{~nm})$ also resulted in a pronounced induction of ROS production linearly correlated to adverse effects, irrespective of the duration of the exposure ( 24 h, 3 days or 8 days). Again, sod-2 and sod-3 mutants were more susceptible than wild-type, with safety concentrations at least 10 times lower. $^{54}$

4.3.3. Metal toxicity. Polak et al. showed evidence for the protective role of metallothioneins and phytochelatin synthase (pcs-1, which produces phytochelatin, a chain of linked glutathione molecules with strong metal-chelating ability) against $30 \mathrm{~nm}$ ZnO-NP. The authors found that NP adverse effects were significantly amplified in a metal sensitive triple knockout mutant ( $m t l-1 ; m t l-2 ; p c s-1)$. They reported transcriptional activation of metallothioneins ( $m t l-1$ and $m t l-2)$, phytochelatin synthase (pcs-1), and an apoptotic marker (cep-1) upon ZnONPs exposure. They demonstrated the oxidative potential of ZnO-NPs in the metal sensitive mutant, and observed significant changes in the nematodes' chemical composition upon ZnO-NPs treatment, suggesting that metallothioneins and phytochelatin synthase are instrumental in the protection against ZnO-NPs induced cytotoxic damage. ${ }^{49}$ Since metallothionein and phytochelatin are critical metal chelators, these results are consistent with ZnO-NPs causing most of their toxicity via dissolution. Many metal ions can cause indirect oxidative stress via inhibition of antioxidant and other enzymes, depletion of glutathione and other antioxidants, or disruption of the electron transport chain.

4.3.4. Controversy over the role of oxidative and metal stress. Not all studies support the role of oxidative stress or metal response as mediators of the toxicity of metal oxide NPs. For instance, Arnold et al. could not attribute $\mathrm{CeO}_{2} \mathrm{NP}$-induced growth inhibition to oxidative or metal stress, but rather proposed a non-specific inhibition of feeding caused by NPs aggregating in the test media and/or inside the gut tract. The authors observed little or no increased sensitivity to $\mathrm{CeO}_{2}-\mathrm{NP}$ exposure in metal (pcs-1, mtl-2) and oxidative stress (sod-3) mutants, hence arguing that there may be only a minor effect of metal or oxidative stress. ${ }^{50}$ Höss et al. also concluded that oxidative stress was not the predominant mechanism of toxicity of soil-derived iron oxide colloids, although sod-2 mutants were more sensitive to some $\mathrm{FeO}_{x}$ and $\mathrm{Fe}^{3+}$ ions. ${ }^{27}$ Interestingly, Gupta et al. found that expression of $m t l-1$ and sod-1 was significantly increased with application of high concentrations of $10 \mathrm{~nm}$ ZnO-NPs, but not significantly affected at lower doses or with 50 and $100 \mathrm{~nm}$, suggesting a non-monotonic doseresponse. $^{48}$

4.3.5. Recent advances in the evaluation of $\mathrm{TiO}_{2}-\mathrm{NPs}$. Among metal oxide nanoparticles, $\mathrm{TiO}_{2}$-NPs is one of the most studied. Their effects on C. elegans have been thoroughly investigated by genetic and metabolic approaches. ${ }^{22,28,109-112}$ Oxidative stress and stress response were identified, however 
only after long exposures and high doses ( $25 \mathrm{mg} \mathrm{L}{ }^{-1}$ for $24 \mathrm{~h}$ ), which suggest the relatively safe properties of $\mathrm{TiO}_{2}$-NPs. ${ }^{28}$ In this line, mutations in genes such as sod-2, sod-3, mtl-2 and hsp-16.48 were identified as conferring susceptibility to the toxicity of engineered $\mathrm{TiO}_{2}$-NPs in C. elegans. ${ }^{113}$ Whole-genome microarray analysis revealed that the regulation of a glutathione- $S$-transferase ( $g s t-3)$, cytochrome $\mathrm{P} 450$ (cypp33-c11), stress resistance regulator (scl-1), oxidoreductase (wah-1) and embryonic development (pod-2) gene were significantly affected by nano- $\mathrm{TiO}_{2} \cdot{ }^{109}$ Ratnasekhar et al. applied metabolomics to identify the biological pathways affected due to $C$. elegans exposure to $\mathrm{TiO}_{2}$-NPs. The authors detected altered regulation of the tricarboxylic acid cycle, arachidonic acid metabolism and glyoxylate dicarboxylate metabolism, which were associated with an effect on reproduction. ${ }^{110}$ These studies demonstrate that the '-omics' disciplines can be applied as a tool to understand the potential toxicity of NPs in a comprehensive manner, including the investigation of organism-environment interactions and molecular effects. ${ }^{110}$

\subsection{Toxicological mechanisms of other nanomaterials}

The cellular and molecular mechanisms of nanotoxicity of other nanomaterials including quantum dots (QD) and carbon nanomaterials such as graphene oxide (GO) have also been addressed recently in $C$. elegans. These studies point out areas that deserve further investigation in the case of the metal and metal oxide NPs. In a study of QD-exposed nematodes, the intestinal barrier played a crucial role in transgenerational toxicity. ${ }^{114}$ Neurotoxic effects via GABAergic neurons and receptors have also been associated with QD treatment. ${ }^{115}$ Among the study of molecular effects, Zhou et al. achieved real-time investigation of QD-induced autophagy in C. elegans and proved that the internalized QDs triggered a complete autophagic process in the intestinal cells. ${ }^{116} \mathrm{Wu}$ et al. found a key role of innate immunity in regulating in vivo GO toxicity. GO toxicity was also found largely dependent on the surface properties of GO, with PEG-modified GO significantly less toxic than pristine GO. ${ }^{112}$ Recently, Subramani et al. investigated the shielding efficacy of BSA and poly ethylene imine (PEI) on graphene oxide (GO) in C. elegans and reported reduced toxicity of BSA-coated GO NPs compared to PEI-coated NPs, confirming the importance of surface properties in particle toxicity in vivo. ${ }^{117}$ Novel genomic studies of GO-treated $C$. elegans have elucidated the importance of long noncoding RNA and microRNAs in the response of this animal model to $\mathrm{GO},{ }^{71,118}$ as well as the importance of specific signaling pathways such as Wnt. ${ }^{119-121}$ Hence, these studies offer a perspective complementary to metal-based NP evaluation, and open new avenues for future research on these materials.

\section{Proposed integrated workflow for biological and materials science-based NP evaluation in $C$. elegans}

Although valuable efforts have been made in the evaluation of metal and metal oxide NPs in C. elegans, the data is heterogeneous and still limited, hampering metadata analysis. To some extent, adoption of the novel methods we describe in this review could exacerbate this heterogeneity. Therefore, to further our understanding of nano/bio interactions and facilitate data integration, we propose adoption of a minimal set of materials science and toxicological assessments that should be included in all studies.

First, it is of vital importance that NPs be very wellcharacterized after their synthesis or their purchase. We propose that the minimum characterization set prior to any biological experiments should include determination of NP size by TEM, hydrodynamic mean diameter by DLS, and surface charge status by ZP (Fig. 16). In addition, colloidal stability of NPs should be investigated in different exposure media well-tolerated by C. elegans. Other experimental parameters (namely, NP concentration range, the developmental stage of C. elegans, the exposure duration and the time-points of study) depend on the objective of the investigation (i.e. ecological $v s$. biomedical, acute $v s$. chronic assay) and should be fixed based on rigorous preliminary experiments.

Similarly, although we presented a wide range of techniques and assays to perform the interaction of nanomaterials with C. elegans, combining materials science and toxicology approaches (Fig. 16), it may not be feasible to apply all the tools proposed either due to limited availability of equipment or time. Therefore, we propose that a minimal set of experiments must be performed consisting of standardized toxicity tests (e.g., $24 \mathrm{~h}$ young adult lethality, $72 \mathrm{~h}$ larval growth, and 3 day reproduction), ${ }^{58}$ which would allow future data meta-analyses. These should be performed in combination with customized experiments to address specific hypothesis/questions on nanotoxicity (i.e. investigation of the recovery responses of acutely and chronically-treated nematodes, ${ }^{44}$ or the effect of NP treatment on C. elegans aging at the molecule, cell, and whole organism level). Finally, from the mechanistic perspective, based on the standing controversy on the role of oxidative stress in nanotoxicity, it is particularly relevant to investigate these mechanisms by complementary techniques (i.e. biochemical assays to detect ROS, use of deletion mutants of antioxidant genes, $\mu$-FTIR spectroscopy).

\section{Conclusions}

In this review, we have presented how toxicology and materials science experts are contributing to better understand nano/bio interactions in the model organism C. elegans, although we have often worked separately, missing joint opportunities between our research fields. We have reviewed the importance of the exposure conditions as a determinant factor of the in vivo effects and toxicity of NPs, and advocate for the adoption of limited protocol standardization in order to gather comparable data, in combination with custom-designed experiments. A major recurrent shortcoming identified is that most of the toxicological studies to date have focused on identifying toxicity endpoints, while very few also investigate NP status inside C. elegans. We believe that a cross-disciplinary approach to address the study of the interactions between inorganic NPs and $C$. elegans would lead to a more solid and comprehensive 
AS SYNTHESIZED NPS / NPS IN THE EXPOSURE MEDIUM

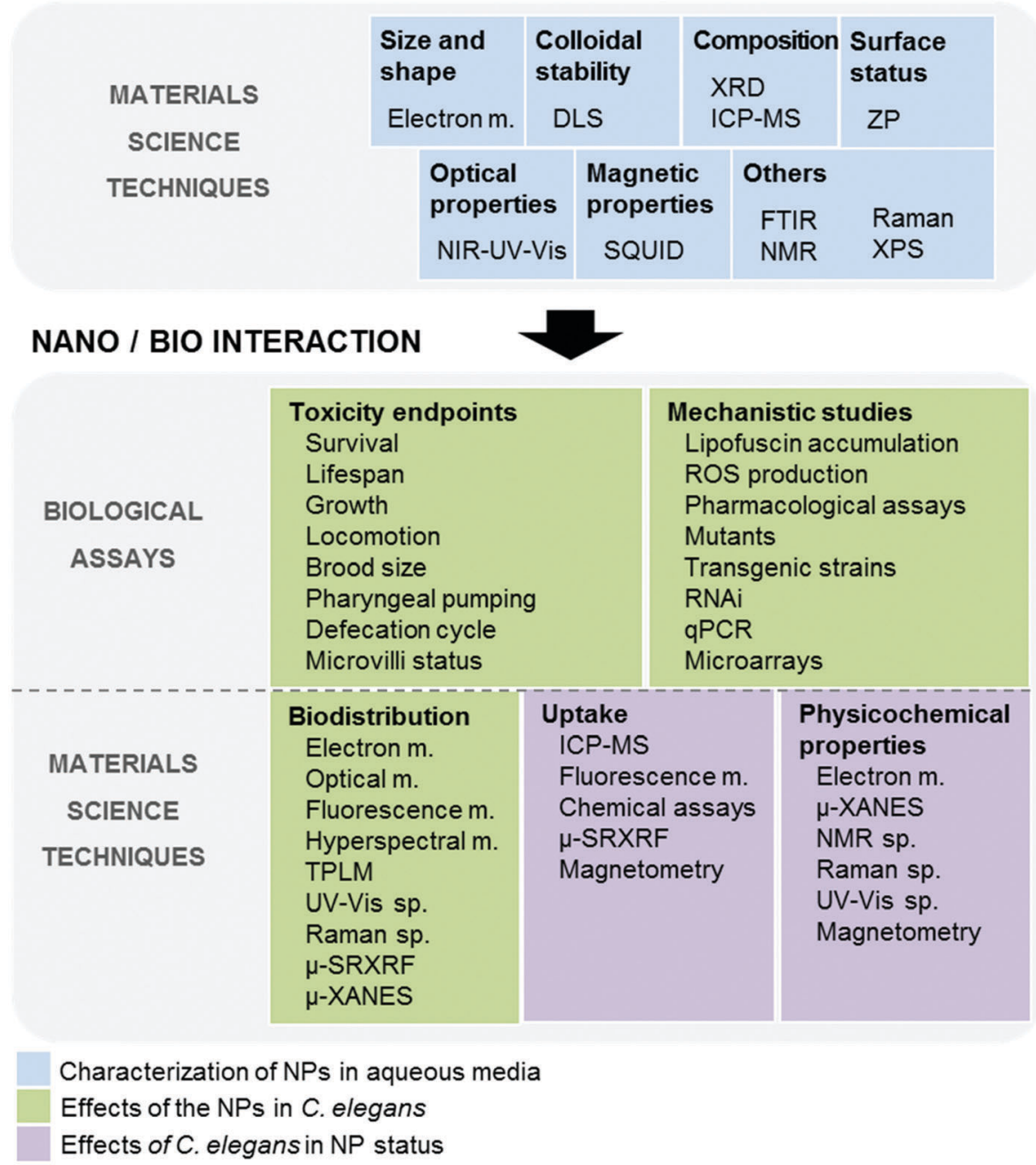

Fig. 16 Proposed techniques and assays to perform a thorough evaluation of nano/bio interactions of NPs in C. elegans combining materials science and toxicology approaches. Legend: $\mathrm{m}$. refers to microscopy, sp. refers to spectroscopy.

evaluation of the two sides of the same coin, and would help to unravel the big picture. Hence, this review was designed to allow the non-expert reader to synergistically combine both research fields to make the experimental results more informative, thorough and robust. For example, from the materials science viewpoint, magnetometry can find a novel use in the study of worms exposed to magnetic NPs; absorbance micro-spectroscopy can be applied to characterize noble metal NPs inside C. elegans; and FT-IR micro-spectroscopy might determine the level of tissue oxidation in NP-treated animals. Current controversial results regarding cell internalization and NP translocation through biological barriers could be addressed combining different imaging techniques to achieve single-particle resolution (i.e. HDFM, TEM, HAADF STEM), together with elemental analysis techniques like EDX or EELS to confirm NP chemical composition and status. From the biological perspective, the analysis of gene expression and the use of specific mutant or transgenic strains could contribute to further the mechanistic understanding of the biological processes involved in nanotoxicity and could potentially guide studies in more complex model organisms. In C. elegans, to date these techniques have revealed NP-induced adverse effects in different cell organelles, among them mitochondria, lysosomes and the nucleus. They have also pointed to the potential role of a variety of molecular mechanisms in response to metal and metal oxide NPs including stress, signaling and metabolic pathways.

As recently highlighted by A. Maynard and R. Aitken, ${ }^{122}$ there is a continuing need to develop better structure-activity relationship (SAR) models describing how engineering nanomaterials interact and perturb biological systems. We advocate that $C$. elegans fulfills the requirements to be exploited as an in vivo platform to perform investigations from the organismal to molecular scales at early stages of nanomaterials' development, integrating the 'safe-by-design' principle approach from the initial discovery phase and providing valuable preliminary data to be integrated in the developing SAR models. However, the appropriate techniques from both toxicology and materials' sides must be combined to thoroughly study nano/bio interactions in C. elegans and extract biologically relevant conclusions 
about NP biocompatibility that may guide/optimize further studies in more complex organisms.

\section{Abbreviations}

\begin{tabular}{|c|c|}
\hline$\mu$-PIXE & Micro-proton-induced X-ray emission \\
\hline$\mu$-SRXRF & Synchrotron radiation X-ray fluorescence \\
\hline BSA & Bovine serum albumin \\
\hline DMSA & Dimercaptosuccinic acid \\
\hline $\mathrm{EC}_{50}$ & Effect concentration $50 \%$ \\
\hline EDX & Energy-dispersive X-ray spectroscopy \\
\hline EELS & Electron energy loss spectroscopy \\
\hline ER & Endoplasmic reticulum \\
\hline FITC & Fluorescein isothiocyanate \\
\hline FT-IR & Fourier transform infrared spectroscopy \\
\hline GO & Graphene oxide \\
\hline HA & Humic acid \\
\hline HAADF & High angle annular dark field \\
\hline HDFM & Hyperspectral dark field microscopy \\
\hline ICP-MS & Inductively coupled plasma mass spectrometry \\
\hline L1 & First C. elegans developmental stage \\
\hline L4 & Last $C$. elegans developmental stage \\
\hline $\mathrm{LC}_{50}$ & Lethal concentration $50 \%$ \\
\hline MHRW & Moderately hard reconstituted water \\
\hline$M_{\mathrm{R}}$ & Remanence magnetization \\
\hline MRI & Magnetic resonance imaging \\
\hline N.S. & Not specified \\
\hline NGM & Nematode growth media \\
\hline NMs & Nanomaterials \\
\hline NPs & Nanoparticles \\
\hline PEG & Polyethylene glycol \\
\hline PEI & Poly ethylene imine \\
\hline PS & Polystyrene \\
\hline PVP & Polyvinylpyrrolidone \\
\hline QD & Quantum dots \\
\hline SEM & Scanning electron microscopy \\
\hline SPIONs & Superparamagnetic iron oxide nanoparticles \\
\hline SQUID & Superconducting quantum interference devices \\
\hline Stage & Developmental stage of $C$. elegans \\
\hline STEM & Scanning transmission electron microscopy \\
\hline STIM & Scanning transmission ion microscopy \\
\hline STORM & Stochastic optical reconstruction microscopy \\
\hline$T_{\mathrm{B}}$ & Blocking temperature \\
\hline TEM & Transmission electron microscopy \\
\hline TPLM & Two-photon luminescence microscopy \\
\hline UV-Vis-NIR & UV-visible-near infrared light \\
\hline XANES & X-ray absorption near edge spectroscopy \\
\hline ZFC-FC & Zero field cooled field cooled \\
\hline
\end{tabular}

\section{Acknowledgements}

This research was partially funded by the Spanish Ministry of Economy and Competitiveness co-funded by European Social Funds (project MAT2015-64442-R, the "Severo Ochoa" Programme for Centres of Excellence in R\&D (SEV-2015-0496),
FPU program (LGM, FPU12/05549)), the Generalitat de Catalunya (2014SGR213), People Program of the European Commission (grant agreement no. 303630, co-funded by the European Social Fund), the COST Action GENIE (Action No. BM1408-A) and the NIH (1R21ES026743 to JNM). This work was also supported by the National Science Foundation (NSF) and the Environmental Protection Agency (EPA) under the NSF Cooperative Agreement EF-0830093, Center for the Environmental Implications of NanoTechnology (CEINT). Any opinions, findings, conclusions, or recommendations expressed in this material are those of the author(s) and do not necessarily reflect the views of the NSF or the EPA. This work has not been subjected to EPA review and no official endorsement should be inferred.

\section{References}

1 Y. Zhao, Q. Wu, Y. Li and D. Wang, RSC Adv., 2013, 3, 5741-5757.

2 L. Gonzalez-Moragas, A. Roig and A. Laromaine, Adv. Colloid Interface Sci., 2015, 219, 10-26.

3 J. Choi, O. V. Tsyusko, J. M. Unrine, N. Chatterjee, J.-M. Ahn, X. Yang, B. L. Thornton, I. T. Ryde, D. Starnes and J. N. Meyer, Environ. Chem., 2014, 11, 227-246.

4 M. C. Leung, P. L. Williams, A. Benedetto, C. Au, K. J. Helmcke, M. Aschner and J. N. Meyer, Toxicol. Sci., 2008, 106, 5-28.

5 D. L. Riddle, T. Blumenthal, B. J. Meyer and J. R. Priess, C. elegans II, Cold Spring Harbor Laboratory Press, Cold Spring Harbor NY, 1997.

6 M. M. Swanson, M. L. Edgley and D. L. Riddle, The nematode Caenorhabditis elegans, 1984.

7 S. E. Hulme and G. M. Whitesides, Angew. Chem., Int. Ed., 2011, 50, 4774-4807.

8 L. L. Maurer, A. L. Luz and J. N. Meyer, in Drug-Induced Mitochondrial Dysfunction, ed. J. A. Dykens and Y. Will, Wiley, 2017.

9 M. Arvanitis, D.-D. Li, K. Lee and E. Mylonakis, Front. Cell. Infect. Microbiol., 2013, 3, 67.

10 V. M. Chauhan, G. Orsi, A. Brown, D. I. Pritchard and J. W. Aylott, ACS Nano, 2013, 7, 5577-5587.

11 S. F. Lim, R. Riehn, W. S. Ryu, N. Khanarian, C. K. Tung, D. Tank and R. H. Austin, Nano Lett., 2006, 6, 169-174.

12 N. Mohan, C.-S. Chen, H.-H. Hsieh, Y.-C. Wu and H.-C. Chang, Nano Lett., 2010, 10, 3692-3699.

13 B. K. Sanches Moraes, S. M. Vieira, W. G. Salgueiro, L. R. Michels, L. M. Colome, D. S. Avila and S. E. Haas, J. Nanosci. Nanotechnol., 2016, 16, 1257-1264.

14 D. Colmenares, Q. Sun, P. Shen, Y. Yue, D. J. McClements and Y. Park, Food Chem., 2016, 202, 451-457.

15 E. Miyako, S. A. Chechetka, M. Doi, E. Yuba and K. Kono, Angew. Chem., Int. Ed., 2015, 54, 9903-9906.

16 H. Huang, S. Delikanli, H. Zeng, D. M. Ferkey and A. Pralle, Nat. Nanotechnol., 2010, 5, 602-606.

17 S. Charan, F. C. Chien, N. Singh, C. W. Kuo and P. Chen, Chemistry, 2011, 17, 5165-5170. 
18 J. H. Kim, S. H. Lee, Y. J. Cha, S. J. Hong, S. K. Chung, T. H. Park and S. S. Choi, Sci. Rep., 2017, 7, 40225.

19 B. P. Gupta and P. Rezai, Micromachines, 2016, 7, 123.

20 R. N. Carvalho, A. Arukwe, S. Ait-Aissa, A. Bado-Nilles, S. Balzamo, A. Baun, S. Belkin, L. Blaha, F. Brion, D. Conti, N. Creusot, Y. Essig, V. E. V. Ferrero, V. Flander-Putrle, M. Furhacker, R. Grillari-Voglauer, C. Hogstrand, A. Jonas, J. B. Kharlyngdoh, R. Loos, A.-K. Lundebye, C. Modig, P.-E. Olsson, S. Pillai, N. Polak, M. Potalivo, W. Sanchez, A. Schifferli, K. Schirmer, S. Sforzini, S. R. Sturzenbaum, L. Softeland, V. Turk, A. Viarengo, I. Werner, S. Yagur-Kroll, R. Zounkova and T. Lettieri, Toxicol. Sci., 2014, 141, 218-233.

21 A. J. Bone, C. W. Matson, B. P. Colman, X. Yang, J. N. Meyer and R. T. Di Giulio, Environ. Toxicol. Chem., 2015, 34, 275-282.

22 Q. Wu, W. Wang, Y. Li, B. Ye, M. Tang and D. Wang, J. Hazard. Mater., 2012, 243, 161-168.

23 J.-y. Roh, S. J. Sim, J. Yi, K. Park, K. H. Chung, D.-y. Ryu and J. Choi, Environ. Sci. Technol., 2009, 43, 3933-3940.

24 H. Ma, P. M. Bertsch, T. C. Glenn, N. J. Kabengi and P. L. Williams, Environ. Toxicol. Chem., 2009, 28, 1324-1330.

25 Y. Gao, N. Liu, C. Chen, Y. Luo, Y. Li, Z. Zhang, Y. Zhao, B. Zhao, A. Iida and Z. Chai, J. Anal. At. Spectrom., 2008, 23, 1121.

26 B. Collin, E. Oostveen, O. V. Tsyusko and J. M. Unrine, Environ. Sci. Technol., 2014, 48, 1280-1289.

27 S. Hoess, A. Fritzsche, C. Meyer, J. Bosch, R. U. Meckenstock and K. U. Totsche, Environ. Sci. Technol., 2015, 49, 544-552.

28 Q. Rui, Y. Zhao, Q. Wu, M. Tang and D. Wang, Chemosphere, 2013, 93, 2289-2296.

29 G. I. Dawlatsina, R. T. Minullina and R. F. Fakhrullin, Nanoscale, 2013, 5, 11761-11769.

30 Y. Qu, W. Li, Y. Zhou, X. Liu, L. Zhang, L. Wang, Y.-f. Li, A. Iida, Z. Tang, Y. Zhao, Z. Chai and C. Chen, Nano Lett., 2011, 11, 3174-3183.

31 J. Kim, M. Takahashi, T. Shimizu, T. Shirasawa, M. Kajita, A. Kanayama and Y. Miyamoto, Mech. Ageing Dev., 2008, 129, 322-331.

32 O. V. Tsyusko, J. M. Unrine, D. Spurgeon, E. Blalock, D. Starnes, M. Tseng, G. Joice and P. M. Bertsch, Environ. Sci. Technol., 2012, 46, 4115-4124.

33 J. N. Meyer, C. A. Lord, X. Y. Yang, E. A. Turner, A. R. Badireddy, S. M. Marinakos, A. Chilkoti, M. R. Wiesner and M. Auffan, Aquat. Toxicol., 2010, 100, 140-150.

34 L. Gonzalez-Moragas, S.-M. Yu, E. Carenza, A. Laromaine and A. Roig, ACS Biomater. Sci. Eng., 2015, 1, 1129-1138.

35 X. Yang, C. Jiang, H. Hsu-Kim, A. R. Badireddy, M. Dykstra, M. Wiesner, D. E. Hinton and J. N. Meyer, Environ. Sci. Technol., 2014, 48, 3486-3495.

36 X. Yang, A. P. Gondikas, S. M. Marinakos, M. Auffan, J. Liu, H. Hsu-Kim and J. N. Meyer, Environ. Sci. Technol., 2012, 46, 1119-1127.

37 L. L. Maurer, X. Yang, A. J. Schindler, R. K. Taggart, C. Jiang, H. Hsu-Kim, D. R. Sherwood and J. N. Meyer, Nanotoxicology, 2016, 10, 831-835.

38 J.-M. Ahn, H.-J. Eom, X. Yang, J. N. Meyer and J. Choi, Chemosphere, 2014, 108, 343-352.
39 D. L. Starnes, J. M. Unrine, C. P. Starnes, B. E. Collin, E. K. Oostveen, R. Ma, G. V. Lowry, P. M. Bertsch and O. V. Tsyusko, Environ. Pollut., 2015, 196, 239-246.

40 D. L. Starnes, S. S. Lichtenberg, J. M. Unrine, C. P. Starnes, E. K. Oostveen, G. V. Lowry, P. M. Bertsch and O. V. Tsyusko, Environ. Pollut., 2016, 213, 314-321.

41 S. W. Kim, J. I. Kwak and Y.-J. An, Environ. Sci. Technol., 2013, 47, 5393-5399.

42 J. Kim, T. Shirasawa and Y. Miyamoto, Biomaterials, 2010, 31, 5849-5854.

43 Q. Le Trequesser, G. Saez, G. Deves, C. Michelet, P. Barberet, M.-H. Delville and H. Seznec, Nucl. Instrum. Methods Phys. Res., Sect. B, 2014, 341, 58-64.

44 Y. Zhao, Q. Wu, M. Tang and D. Wang, Nanomedicine, 2014, 10, 89-98.

45 Q. Wu, A. Nouara, Y. Li, M. Zhang, W. Wang, M. Tang, B. Ye, J. Ding and D. Wang, Chemosphere, 2013, 90, 1123-1131.

46 H. Wang, R. L. Wick and B. Xing, Environ. Pollut., 2009, 157, 1171-1177.

47 J. Y. Roh, Y. K. Park, K. Park and J. Choi, Environ. Toxicol. Pharmacol., 2010, 29, 167-172.

48 S. Gupta, T. Kushwah, A. Vishwakarma and S. Yadav, Nanoscale Res. Lett., 2015, 10, 1010.

49 N. Polak, D. S. Read, K. Jurkschat, M. Matzke, F. J. Kelly, D. J. Spurgeon and S. R. Stuerzenbaum, Comp. Biochem. Physiol., Part C: Toxicol. Pharmacol., 2014, 160, 75-85.

50 M. C. Arnold, A. R. Badireddy, M. R. Wiesner, R. T. Di Giulio and J. N. Meyer, Arch. Environ. Contam. Toxicol., 2013, 65, 224-233.

51 A. Scharf, A. Piechulek and A. von Mikecz, ACS Nano, 2013, 7, 10695-10703.

52 A. Pluskota, E. Horzowski, O. Bossinger and A. von Mikecz, PLoS One, 2009, 4, e6622.

53 Y. Li, S. Yu, Q. Wu, M. Tang, Y. Pu and D. Wang, J. Hazard. Mater., 2012, 219-220, 221-230.

54 Q. Wu, Y. Li, M. Tang and D. Wang, PLoS One, 2012, 7, e43729.

55 Y. Zhao, Q. Wu, Y. Li, A. Nouara, R. Jia and D. Wang, Nanoscale, 2014, 6, 4275-4284.

56 Q. Wang, Y. Zhou, B. Song, Y. Zhong, S. Wu, R. Cui, H. Cong, Y. Su, H. Zhang and Y. He, Small, 2016, 12, 3143-3154.

57 T. Stiernagle, Wormbook, DOI: 10.1895/wormbook.1.101.1. 58 L. L. Maurer, I. T. Ryde, X. Yang and J. N. Meyer, Current Protocols in Toxicology, John Wiley \& Sons, Inc., 2015, DOI: 10.1002/0471140856.tx2010s66.

59 D. Lim, J.-y. Roh, H.-j. Eom, J.-Y. Choi, J. Hyun and J. Choi, Environ. Toxicol. Chem., 2012, 31, 585-592.

60 L. Ellegaard-Jensen, K. A. Jensen and A. Johansen, Ecotoxicol. Environ. Saf., 2012, 80, 216-223.

61 E. Q. Contreras, H. L. Puppala, G. Escalera, W. Zhong and V. L. Colvin, Environ. Toxicol. Chem., 2014, 33, 2716-2723.

62 J.-Y. Roh, H.-J. Eom and J. Choi, Toxicol. Res., 2012, 28, 19-24.

63 S. W. Kim, S.-H. Nam and Y.-J. An, Ecotoxicol. Environ. Saf., 2012, 77, 64-70. 
64 L. Gonzalez-Moragas, P. Berto, C. Vilches, R. Quidant, A. Kolovou, R. Santarella-Mellwig, Y. Schwab, S. Stürzenbaum, A. Roig and A. Laromaine, Acta Biomater., 2017, 53, 598-609.

65 N. Khanna, C. P. Cressman, 3rd, C. P. Tatara and P. L. Williams, Arch. Environ. Contam. Toxicol., 1997, 32, 110-114.

66 S. G. Donkin and P. L. Williams, Environ. Toxicol. Chem., 1995, 14, 2139-2147.

67 L. Gonzalez-Moragas, PhD in Materials Science Doctoral thesis, Universitat Autònoma de Barcelona, 2016.

68 S.-M. Yu, L. Gonzalez-Moragas, M. Milla, A. Kolovou, R. Santarella-Mellwig, Y. Schwab, A. Laromaine and A. Roig, Acta Biomater., 2016, 43, 348-357.

69 D. Wang, Toxicol. Res., 2016, 5, 1003-1011.

70 Q. Wu, L. Yin, X. Li, M. Tang, T. Zhang and D. Wang, Nanoscale, 2013, 5, 9934-9943.

71 Q. Wu, X. Zhou, X. Han, Y. Zhuo, S. Zhu, Y. Zhao and D. Wang, Biomaterials, 2016, 102, 277-291.

72 C. Fang-Yen, L. Avery and A. D. T. Samuel, Proc. Natl. Acad. Sci. U. S. A., 2009, 106, 20093-20096.

73 Q. Wu, Y. Li, Y. Li, Y. Zhao, L. Ge, H. Wang and D. Wang, Nanoscale, 2013, 5, 11166-11178.

74 L. Gonzalez-Moragas, S.-M. Yu, N. Benseny-Cases, S. Stürzenbaum, A. Roig and A. Laromaine, 2017, submitted.

75 I. Kolotuev, D. J. Bumbarger, M. Labouesse and Y. Schwab, Methods Cell Biol., 2012, 111, 203-222.

76 S. Hoess, K. Schlottmann and W. Traunspurger, Environ. Sci. Technol., 2011, 45, 10219-10225.

77 B. P. Jackson, P. L. Williams, A. Lanzirotti and P. M. Bertsch, Environ. Sci. Technol., 2005, 39, 5620-5625.

78 M. E. Johnson, S. K. Hanna, A. R. Montoro Bustos, C. M. Sims, L. C. C. Elliott, A. Lingayat, A. C. Johnston, B. Nikoobakht, J. T. Elliott, R. D. Holbrook, K. C. K. Scott, K. E. Murphy, E. J. Petersen, L. L. Yu and B. C. Nelson, ACS Nano, 2017, 11, 526-540.

79 M. R. Beversluis, A. Bouhelier and L. Novotny, Phys. Rev. B: Condens. Matter Mater. Phys., 2003, 68, 115433.

80 P. Ghenuche, S. Cherukulappurath, T. H. Taminiau, N. F. van Hulst and R. Quidant, Phys. Rev. Lett., 2008, 101, 116805.

81 J. P. Wilcoxon, J. E. Martin, F. Parsapour, B. Wiedenman and D. F. Kelley, J. Chem. Phys., 1998, 108, 9137-9143.

82 M. B. Mohamed, V. Volkov, S. Link and M. A. El-Sayed, Chem. Phys. Lett., 2000, 317, 517-523.

83 N. Gao, Y. Chen, L. Li, Z. Guan, T. Zhao, N. Zhou, P. Yuan, S. Q. Yao and Q.-H. Xu, J. Phys. Chem. C, 2014, 118, 13904-13911.

84 J. Chen, C. Guo, M. Wang, L. Huang, L. Wang, C. Mi, J. Li, X. Fang, C. Mao and S. Xu, J. Mater. Chem., 2011, 21, 2632.

85 E. Carenza, V. Barcelo, A. Morancho, J. Montaner, A. Rosell and A. Roig, Acta Biomater., 2014, 10, 3775-3785.

86 R. T. Minullina, Y. N. Osin, D. G. Ishmuchametova and R. F. Fakhrullin, Langmuir, 2011, 27, 7708-7713.

87 D. Ami, A. Natalello, A. Zullini and S. M. Doglia, FEBS Lett., 2004, 576, 297-300.

88 B. Aksu, Marmara Pharm. J., 2015, 19, 12-18.
89 N. D. Mathew, M. D. Mathew and P. P. T. Surawski, Anal. Biochem., 2014, 450, 52-56.

90 C. D. Walkey, J. B. Olsen, F. Song, R. Liu, H. Guo, D. W. H. Olsen, Y. Cohen, A. Emili and W. C. W. Chan, ACS Nano, 2014, 8, 2439-2455.

91 P. Chen, E. J. Martinez-Finley, J. Bornhorst, S. Chakraborty and M. Aschner, Front. Aging Neurosci., 2013, 5, 18.

92 S. A. James, M. D. de Jonge, D. L. Howard, A. I. Bush, D. Paterson and G. McColl, Metallomics, 2013, 5, 627-635.

93 W. H. Li, F. L. Hsu, J. T. Liu and V. H. Liao, Food Chem. Toxicol., 2011, 49, 812-819.

94 M. Roman, P. Jitaru and C. Barbante, Metallomics, 2014, 6, 25-54.

95 K. Ganio, S. A. James, D. J. Hare, B. R. Roberts and G. McColl, Analyst, 2016, 141, 1434-1439.

96 R. Dhawan, D. B. Dusenbery and P. L. Williams, Environ. Toxicol. Chem., 2000, 19, 3061-3067.

97 R. Dhawan, D. B. Dusenbery and P. L. Williams, J. Toxicol. Environ. Health, Part A, 1999, 58, 451-462.

98 J. Liu and R. H. Hurt, Environ. Sci. Technol., 2010, 44, 2169-2175.

99 X. Luo, S. Xu, Y. Yang, L. Li, S. Chen, A. Xu and L. Wu, Sci. Rep., 2016, 6, 36465.

100 M. Levy, F. Lagarde, V. A. Maraloiu, M. G. Blanchin, F. Gendron, C. Wilhelm and F. Gazeau, Nanotechnology, 2010, 21, 395103.

101 S. J. Soenen, P. Rivera-Gil, J.-M. Montenegro, W. J. Parak, S. C. De Smedt and K. Braeckmans, Nano Today, 2011, 6, 446-465.

102 S. J. Soenen, W. J. Parak, J. Rejman and B. Manshian, Chem. Rev., 2015, 115, 2109-2135.

103 S. Sabella, R. P. Carney, V. Brunetti, M. A. Malvindi, N. Al-Juffali, G. Vecchio, S. M. Janes, O. M. Bakr, R. Cingolani, F. Stellacci and P. P. Pompa, Nanoscale, 2014, 6, 7052-7061.

104 B. D. Gaytan and C. D. Vulpe, Front. Genet., 2014, 5, 110.

105 D. A. Basketter, H. Clewell, I. Kimber, A. Rossi, B. Blaauboer, R. Burrier, M. Daneshian, C. Eskes, A. Goldberg, N. Hasiwa, S. Hoffmann, J. Jaworska, T. B. Knudsen, R. Landsiedel, M. Leist, P. Locke, G. Maxwell, J. McKim, E. A. McVey, G. Ouedraogo, G. Patlewicz, O. Pelkonen, E. Roggen, C. Rovida, I. Ruhdel, M. Schwarz, A. Schepky, G. Schoeters, N. Skinner, K. Trentz, M. Turner, P. Vanparys, J. Yager, J. Zurlo and T. Hartung, Altex, 2012, 29, 3-91.

106 A. Asati, S. Santra, C. Kaittanis and J. M. Perez, ACS Nano, 2010, 4, 5321-5331.

107 Z. Savoly, G. Buzanich, G. Pepponi, C. Streli, K. Hracs, P. I. Nagy and G. Zaray, Microchem. J., 2015, 118, 80-87.

108 S. Rocheleau, M. Arbour, M. Elias, G. I. Sunahara and L. Masson, Nanotoxicology, 2015, 9, 502-512.

109 S. Rocheleau, M. Arbour, M. Elias, G. I. Sunahara and L. Masson, Nanotoxicology, 2015, 9, 502-512.

110 C. Ratnasekhar, M. Sonane, A. Satish and M. K. R. Mudiam, Nanotoxicology, 2015, 9, 994-1004.

111 J.-Y. Roh, Y.-K. Park, K. Park and J. Choi, Environ. Toxicol. Pharmacol., 2010, 29, 167-172.

112 Q. Wu, Y. Zhao, J. Fang and D. Wang, Nanoscale, 2014, 6, 5894-5906. 
113 Q. Wu, Y. Zhao, Y. Li and D. Wang, Nanomedicine, 2014, 10, 1263-1271.

114 Z. Liu, X. Zhou, Q. Wu, Y. Zhao and D. Wang, RSC Adv., 2015, 5, 94257-94266.

115 Y. Zhao, X. Wang, Q. Wu, Y. Li, M. Tang and D. Wang, Toxicol. Res., 2015, 4, 399-408.

116 Y. Zhou, Q. Wang, B. Song, S. Wu, Y. Su, H. Zhang and Y. He, Biomaterials, 2015, 72, 38-48.

117 S. Subramani, S. Govindan, P. Sundararaj, S. L. Hafez, P. Nagamony and N. Sundararaj, J. Nematol., 2016, 48, 374.
118 Q. Wu, Y. Zhao, G. Zhao and D. Wang, Nanomedicine, 2014, 10, 1401-1410.

119 N. Chatterjee, Y. Kim, J. Yang, C. P. Roca, S.-W. Joo and J. Choi, Nanotoxicology, 2017, 11, 76-86.

120 L. Zhi, M. Qu, M. Ren, L. Zhao, Y. Li and D. Wang, Carbon, 2017, 113, 122-131.

121 L. Zhi, M. Ren, M. Qu, H. Zhang and D. Wang, Sci. Rep., 2016, 6, 39261.

122 A. D. Maynard and R. J. Aitken, Nat. Nanotechnol., 2016, 11, 998-1000. 